\title{
Optimized Activation for Quantum-Inspired Self-supervised Neural Network based Fully Automated Brain MR Image Segmentation
}

This paper was downloaded from TechRxiv (https://www.techrxiv.org).

\section{LICENSE}

CC BY 4.0

\section{SUBMISSION DATE / POSTED DATE}

02-09-2020 / 04-09-2020

\section{CITATION}

Konar, Debanjan; Bhattacharyya, Siddhartha; Panigrahi, Bijaya Ketan (2020): Optimized Activation for Quantum-Inspired Self-supervised Neural Network based Fully Automated Brain MR Image Segmentation. TechRxiv. Preprint. https://doi.org/10.36227/techrxiv.12909872.v1

$\mathrm{DOI}$ 


\title{
Optimized Activation for Quantum-Inspired Self-supervised Neural Network based Fully Automated Brain MR Image Segmentation
}

\author{
Debanjan Konar ${ }^{\mathrm{a}}$, Siddhartha Bhattacharyya ${ }^{\mathrm{b}, *}$, Sandip Dey ${ }^{\mathrm{c}}$, Bijaya Ketan \\ Panigrahi $^{\mathrm{a}}$ \\ ${ }^{a}$ Department of Electrical Engineering, Indian Institute of Technology Delhi, New Delhi, \\ India \\ ${ }^{b}$ Department of Computer Science and Engineering, CHRIST (Deemed to be University), \\ Bangalore, India \\ ${ }^{c}$ Department of Computer Science, Sukanta Mahavidyalaya, Jalpaiguri, India
}

\begin{abstract}
The slow-convergence problem degrades the segmentation performance of the recently proposed Quantum-Inspired Self-supervised Neural Network models owing to lack of suitable tailoring of the inter-connection weights. Hence, incorporation of quantum-inspired meta-heuristics in the Quantum-Inspired Selfsupervised Neural Network models optimizes their hyper-parameters and interconnection weights. This paper is aimed at proposing an optimized version of a Quantum-Inspired Self-supervised Neural Network (QIS-Net) model for optimal segmentation of brain Magnetic Resonance (MR) Imaging. The suggested Optimized Quantum-Inspired Self-supervised Neural Network (Opti-QISNet) model resembles the architecture of QIS-Net and its operations are leveraged to obtain optimal segmentation outcome. The optimized activation function employed in the presented model is referred to as Quantum-Inspired Optimized Multi-Level Sigmoidal (Opti-QSig) activation. The Opti-QSig activation function is optimized by three quantum-inspired meta-heuristics with fitness evaluation using Otsu's multi-level thresholding. Rigorous experiments have been conducted on Dynamic Susceptibility Contrast (DSC) brain MR images from Nature data repository. The experimental outcomes show that the proposed self-supervised Opti-QISNet model offers a promising alternative to the deeply supervised neural network based architectures (UNet and FCNNs) in medical image segmentation and outperforms our recently developed models QIBDS Net and QIS-Net.
\end{abstract}

Keywords: Quantum Computing, MR images, QIS-Net, U-Net

\footnotetext{
${ }^{*}$ Corresponding author

Email address: dr.siddhartha.bhattacharyya@gmail.com (Siddhartha Bhattacharyya)
} 


\section{Introduction}

The brain is formidably most complex entity in human body. Recent years have witnessed the rapid increase in brain cancer diseases owing to abnormal cell divisions and unnatural growth of the cells in human brain. To obtain better prognosis, early diagnosis of brain abnormalities such as degenerative, infectious, ischemic or malignant is preferred. The essential key information (shape, size and orientation) relevant to MR image segmentation are required for diagnosis of brain tumour. However, manual segmentation of brain tumour is a paramount task for the radiologist due to low image resolution, variation in shape, size, orientation, imaging protocols and inter-observer and intra-observer variability. In addition, infiltration of brain tissues prone to mass effect and overlapping are the most challenging characteristics of brain tumor segmentation. In turn, automated segmentation approaches have gained wide acceptance among computer vision researchers owing to precise and robust segmentation [1, 2]. A plethora of approaches has been already entrusted targeting automatic MR image segmentation using supervised and unsupervised learning [3]. In contrast to automated image segmentation, Convolutional Neural Networks (CNN) based deep learning techniques [4] have received much attention due to their state-ofthe-art performance. However, these methods suffer from lack of generalization and image specific adaptation.

The traditional classical computing paradigms face various challenges and are also not efficient to solve complex computational problems in engineering domain. Quantum computing and quantum information processing rely on the quantum mechanical phenomena and also explore a new horizon of research in the field of computer science to solve those uphill tasks which are highly time consuming [5]. The new emerging quantum-inspired computationally complex systems $[6,7,8]$ have been found to outperform the classical systems efficiently. In the field of computer vision, quantum-inspired neural networks (QINN) $[9,10,11,12,13,14,15]$ received attention significantly for solving problems relevant to pattern recognition and classification exploiting the inherent parallelism offered by qubits. However, time intensive and computationally complex quantum back-propagation algorithms are employed in the supervised quantum-inspired neural network models for training. Moreover, QINN models fall short of the multi-level activation function and consequently, they fail to produce optimal thresholding for multi-level images. Recently, quantuminspired neural networks referred to as QIBDS-Net and QIS-Net architectures are suggested by Konar et al. $[16,17]$ for fully automated brain MR image segmentation. QIBDS-Net [16] and QIS-Net [17] fail to provide optimal outcome owing to wide variation of gray-scales in brain MR images and often suffer from convergence problems. The key aim of this current work is to develop an optimized version of our previously developed quantum-inspired self-supervised neural network models $[16,17]$ for fully automated medical image segmentation without any form supervision or training. The significant contributions of the proposed work are as follows.

1. The suggested Opti-QISNet model primarily focuses on the activation 
function characterization exhibiting adaptive threshold schemes via Otsu's multi-level thresholding [18]. It is optimized by trinity of quantum-inspired meta heuristics developed in our previous work [19] (Quantum-inspired Differential Evolution (QDE), Quantum-inspired Ant Colony Optimization (QACO) and Quantum-inspired Particle Swarm Optimization (QPSO)).

2. In addition, the image pixel intensity dependent adaptive optimized thresholding governs the tuning of the hyper parameters associated with our suggested optimized network model. The optimization procedure allows the quantum-inspired activation function to replicate for different gray scales present in the underlying image with adaptive step size as fitness evaluated by the meta-heuristics and hence yields optimal results.

3. The convergence of our suggested Opti-QISNet model is also analysed mathematically to prove its super-linearity along with experimental demonstration. Our proposed neural network model is guided by the optimized inter-connection weights, thereby enabling optimal segmented tumor regions.

It may be noted that this manuscript is an extended version of the preliminary work (Opti-QIBDS Net) [20] and substantial revision work has been accomplished on Opti-QIBDS Net. The primary technical distinction of the current work lies in the fact that Opti-QISNet involves trinity of quantum-inspired meta-heuristics for optimizing the thresholds in the optimized quantum-inspired activation function incorporating Otsu's multi-level thresholding as the fitness function. In addition to this, the modification of the conference paper is accomplished by elaborating the proposed Opti-QISNet model, extensive literature reviews, convergence analysis of the network operations and including more experimental results.

The remainder section of the article is reported as follows: Section 2 discusses a compact literature review pertaining to image segmentation of MR images. Introduction to quantum computing and the key concepts relevant to the current quantum inspired neural network (QNN) are elucidated in Section 3. Three quantum-inspired meta-heuristic algorithms employed in the optimization process of the suggested network are illustrated in Section 4. The proposed optimized activation function with Quantum-Inspired Self-supervised Neural Network (QIS-Net) architecture and its principle of operation are explained vividly in Section 5. The network weight optimization and the convergence analysis of the Opti-QISNet are reflected in Section 6. Rigorous experimental results and discussions are presented in Section 7. Finally, conclusive remarks and future directions of research are confabulated in Section 8.

\section{Related Work}

In the field of computer vision and medical image analysis, Artificial Neural Networks (ANNs) have received considerable attention among the research community owing to inherent non-linear and adaptive computing capabilities [21, 22, 23]. A knowledge based fast and efficient 2D MR image segmentation using 
a constraint satisfied Boolean neural network [24] is a notable example in this direction. In addition, an automatic segmentation of brain lesions on MR images is contributed by Zikic et al. [25] guided by fuzzy-logic inspired neural network architecture. Bauer et al. [26] also suggested a fully automatic delineation of brain tumour boundaries combining the multi-spectral intensity assisted support vector machine classifier followed by conditional random field guided hierarchical regularization. A probabilistic neural network (PNN) classifier characterized by the second degree nonlinear least squares features transformation (LSFT) is introduced by Georgiadis et al. [21] for Dynamic Susceptibility Contrast (DSC) Brain MR image segmentation. Kumar et al. [22] also presented a boundary based Gradient Vector Flow (GVF) approach for region of interest in MR images and subsequently performed multi-class classification using a Principal Component Analysis-Artificial neural network (PCA-ANN) classifier. A popular supervised Self-Organizing Map (SOM) followed by probability based clustering by Ortiz et al. [27] is employed for Brain MR image segmentation to enhance the resolution of the segmented images. Nevertheless, these supervised artificial neural network architectures often suffer from the limited feature information and explicitly rely on pixel intensities, hence producing inaccurate segmentation.

Recent years have witnessed the state-of-the-art performance achieved using Convolutional Neural Networks (CNN) in the field of computer vision and medical image analysis [4, 28]. Brain MR image segmentation using CNNs has demonstrated accurate and robust results owing to learning of implicitly relevant and increasingly higher level features. Havaei et al. [29] contributed a novel cascaded CNN architecture exploiting both local and global features tailored to brain tumour segmentation. A modified fully convolutional neural network (UNet) is suggested by Lai et al. [30] incorporating Gaussian-Dirichlet mixture model (GMMD) for brain MR image segmentation. A 2D CNN is proposed by Lyksborg et al. [31] for binary classification of MR images and identification of complete tumour. Of late, Pereira et al. [32] suggested a modified CNN exploiting small size kernels $(3 \times 3)$ to obviate the effect of over fitting due to structural and spatial variations of MR images. Recently, U-Net [28] architecture gained much popularity in 2D slice MR image segmentation. In spite of growing popularity among the computer vision researchers for medical image segmentation, deep learning based network architectures falls short owing to lack of image specific adaptation and lack of expert domain image analysts for labeling of the training images. It also suffers from huge time and space complexity. In addition, deep learning based technologies require extensive computational resources to perform the training.

With the advancement of quantum computing, Quantum-Inspired Neural Networks (QINNs) have gained considerable popularity among the quantum computing researchers in recent times. Kak et al. [33] first proposed the concept of quantum neural network by replicating the classical neural network in quantum formalism which relies on quantum dynamics. Purushothaman et al. [34] also suggested a novel quantum neural network model characterized by multi-level hidden neurons to harness the inherent characteristics of superposition of quan- 
tum states. Of late, a shallow quantum perceptron model with only a single layer has been developed by Kouda et al. [35] for image compression.

It is also worth noting that quantum-inspired neural networks (QINN) rely on the real values of the complex qubits and are implemented on classical systems. However, QINN models exhibit quantum superposition and entanglement properties of quantum computation and therein offer faster computation. The quantum gates are also operated on real angles of phase shift. The controlledHadamard gate assisted quantum-inspired neural networks by Li et al. [24] deserves special mention. Lately, Matusi et al. [36] employed single and two qubit rotation gates in the proposed quantum-inspired network model. Recently, Bhattacharyya et al. $[9,11]$ and Konar et al. $[12,13]$ also contributed a Quantum Multi-Layer Self-Organizing Neural Network (QMLSONN) architecture and Quantum Bidirectional Self-Organizing Neural Network (QBDSONN) architecture, respectively by introducing a single qubit rotation gate. These self-supervised network architectures with bi-level activation are efficient for fast and precise binary image segmentation and also outperform their classical counterparts referred to as multi-layer self organizing neural network (MLSONN) [37] architecture and Bi-Directional Self-Organizing Neural Network (BDSONN) architecture [38], respectively. However, quantum inspired neural network (QINN) frameworks and QMLSONN are guided by a complex quantum back-propagation algorithm [39]. The QBDSONN architecture proposed by Konar et. al [12, 13] obviates the quantum-back-propagation algorithm and resorts to counter-propagation of network patterns. Recently, Konar et al. suggested few quantum-inspired neural network models $[16,17]$ for fully self-supervised brain MR image segmentation. These quantum-inspired selfsupervised neural network models suffer from relatively slow convergence problems while applied on brain MR images with large variations of gray-scales. In this paper, the authors propose an optimized version of the quantum-inspired neural network model referred to as Opti-QISNet which is a novel attempt for fully automatic and optimal segmentation of brain MR images without any external supervision.

\section{Basic Concepts of Quantum Computing}

Implementation of quantum algorithms relies on the basic principles of quantum mechanics like superposition, coherence, decoherence, entanglement [40] and the basic states of quantum computing (qubits or quantum bits) are characterized using these principles.

\subsection{Concept of Qubits}

The quantum bit or qubit [41] is the constituent unit of processing in quantum computing and exists in Hilbert space with superposition of eigenstates $|0\rangle$ and $|1\rangle$ as

$$
|\phi\rangle=\alpha|0\rangle+\beta|1\rangle=\left[\begin{array}{l}
\alpha \\
\beta
\end{array}\right]
$$


subject to normalization criteria

$$
|\alpha|^{2}+|\beta|^{2}=1
$$

where $|\alpha|^{2}$ and $|\beta|^{2}$ corresponds to the probabilities of eigenstates $|0\rangle$ and $|1\rangle$, respectively.

A set of qubits $\left|\phi_{j}\right\rangle$ residing in a Hilbert space with superposition can describe a quantum system using the following wave function [42].

$$
|\psi\rangle=\sum_{j}^{n} p_{j}\left|\phi_{j}\right\rangle
$$

where $p_{j}$ is a complex entity.

The quantum system $|\psi\rangle$ collapses to one of the basis states on interaction with the environment often known as quantum observation [5] subjected to the condition $\sum_{j}^{n}\left|p_{j}\right|^{2}=1$.

\subsection{Quantum Logic Gates}

The quantum logic gates are realized by a series of unitary transformations of quantum bits and forms the basis for quantum information processing. The phase-shift controlled NOT (CNOT) gate and rotation gate are suitable for single or small number of qubits due to reversible behavior. The CNOT gate is defined as [43]

$$
\delta\left(\omega_{1}+\omega_{2}\right)=\delta\left(\omega_{1}\right) \delta\left(\omega_{2}\right)
$$

where $\delta(\omega)=e^{i \omega}=\cos \omega+i \sin \omega$ and $i$ is an imaginary . The CNOT gate with phase shift is defined as

$$
\delta\left(\frac{\pi}{2} \alpha-\omega\right)=\left\{\begin{array}{l}
\cos \omega-i \sin \omega(\alpha=0) \\
\sin \omega+i \cos \omega(\alpha=1)
\end{array}\right.
$$

Another phase shift gate is the quantum rotation gate represented as

$$
R(\omega)=\left[\begin{array}{cc}
\cos \omega & -\sin \omega \\
\sin \omega & \cos \omega
\end{array}\right]
$$

Considering a single quantum bit defined as $|\phi\rangle=\left[\begin{array}{c}\cos \omega_{0} \\ \sin \omega_{0}\end{array}\right]$, the rotation gate $R(\omega)$ shifts $|\phi\rangle$ with an angle $\omega_{0}$ as

$$
R(\omega)|\phi\rangle=\left[\begin{array}{cc}
\cos \omega & -\sin \omega \\
\sin \omega & \cos \omega
\end{array}\right] \times\left[\begin{array}{c}
\cos \omega_{0} \\
\sin \omega_{0}
\end{array}\right]
$$

\section{Quantum-Inspired Meta-Heuristic Algorithms}

\subsection{Quantum-Inspired Ant Colony Optimization}

Ant Colony Optimization (ACO) is a very popular, well admired and efficient population-based technique introduced by Dorigo et al. [44]. They observed and 
studied the behaviour of real ants for struggling for food and applied their experience to develop such an optimization technique in algorithmic form. This technique performs very efficiently when applied to solve different optimization problems. In real life, ants search for food in every possible place. They travel across different paths for this purpose. In their way, they squirt pheromone, a very well known chemical from their body. The objective is to exchange information among each other to discover the shortest possible path to reach at the food source. It is obvious that a specific path comprising more amount of chemical substance is followed by more ants. The motivation of their communal behavior has inspired number of researchers to develop several algorithms that can handle different combinatorial optimization problems [45].

The Quantum Inspired Ant Colony Optimization (QACO), proposed by Dey et al. [19], can efficiently find the optimal threshold values in multi-level thresholding. The authors have successfully applied the proposed technique on gray scale images, synthetic images and colour images to find the optimal thresholds in bi-level/multi-level frameworks [19, 46, 47]. The authors have used the fundamentals of quantum computing like qubits, superposition and other related properties in the backbone of some popular meta-heuristics to form suitable algorithmic structure. In QACO, a population $(P)$ of size $M$, each of length $\mathcal{L}$, has been created as the initial step. A pheromone matrix $(\tau)$ having the identical size of the population, has also been introduced. Along with different steps followed in QACO, the population matrix is updated at every generation using the pheromone matrix as shown in Algorithm 1.

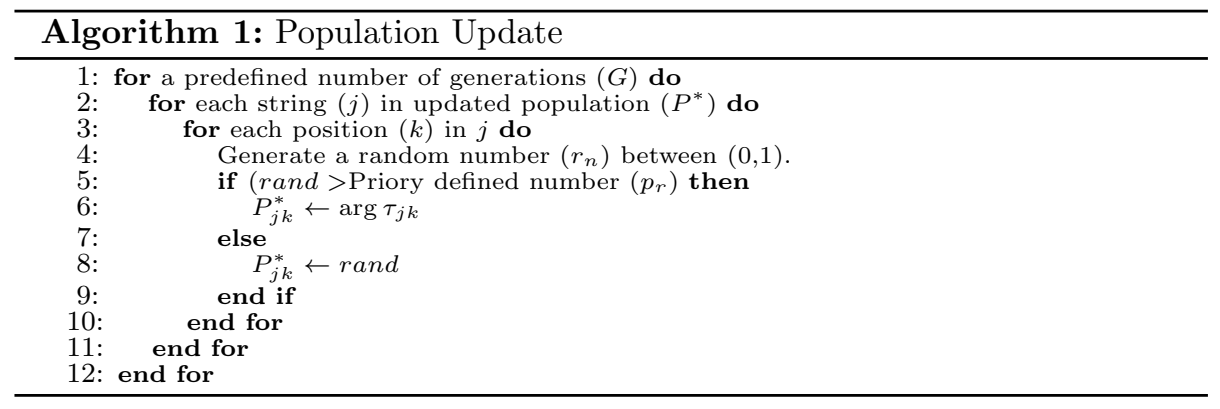

At each generation, $\tau$ is updated using following equation.

$$
\tau_{l m}=(1-\rho) \tau_{l m}+\epsilon \tau_{l m}
$$

where, $\tau_{l m}$ signifies the deposited amount of pheromone. $\rho$ is referred to as the evaporation rate of pheromone. Details regarding this algorithm are available in [19].

\subsection{Quantum-Inspired Differential Evolution}

Storn and Prince introduced a very efficient and well admired stochastic optimization technique known as Differential Evolution (DE) [48]. Due its simplicity, almost hazard free implementation capability, efficiency with reference 
to convergence capability, DE has become an automatic choice for many researchers. One of the major advantages of using DE is that it generally gives remarkable results with very few parameter adjustment. Like QACO, Quantum Inspired Differential Evolution (QDE) proposed by Dey et al. [19], is able to find the optimal thresholds in multi-level thresholding. This technique has also been successfully applied on different gray scale images, synthetic images and colour images. QDE works in a similar fashion as discussed in QACO. At each generation, the population of QDE is successively updated using two genetic operators, called mutation and crossover, as shown in Algorithms 2 and 3.

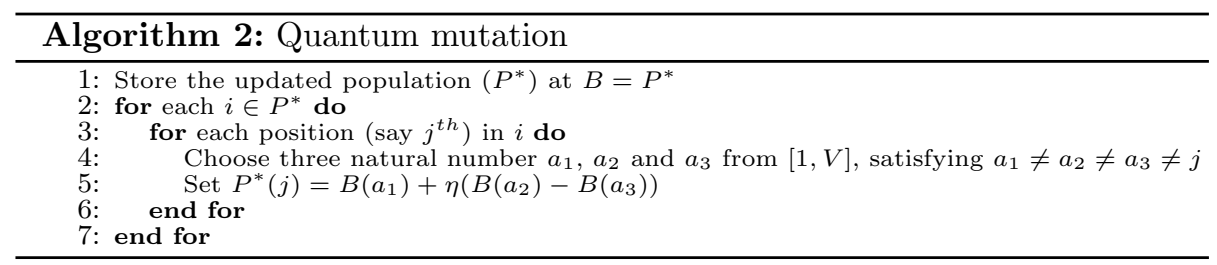

In Algorithm 2, $V$ denotes the number of strings in the populations. In

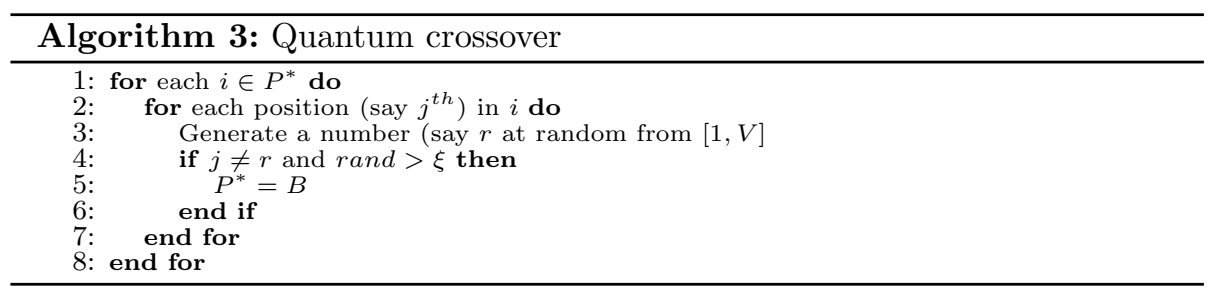

Algorithm 3, $V$ is the number of strings in the populations and $\xi$ represents a predefined crossover probability. Details regarding the QDE algorithm are given in [19].

\subsection{Quantum-Inspired Particle Swarm Optimization}

Kennedy and Eberhart [49] observed and examined the gathering of birds' behavior, which inspired them to develop a popular optimization technique, called Particle Swarm Optimization (PSO). It is observed that birds always try to locate for some search space, and they are habituated to fly within this particular zone. They generally choose that particular paths for visiting, which have already been used by them. Dey et al. [19] have proposed a popular quantum inspired meta-heuristic technique, called Quantum Inspired Particle Swarm Optimization (QPSO). Like the above mentioned techniques, QPSO also exploits the fundamentals of quantum computing and the framework of PSO to combine them to form the proposed technique [19]. The proposed technique has also been applied on same types of images in multi-level domain. At each generation the population $(P)$ of particles $\left(p_{i}\right)$ is updated for population 
diversity.

For each $\left(p_{i}\right) \in P$, the velocity is updated by using the formula given by

$$
v_{i}(g+1)=\varsigma v_{i}(g)+l_{1} d_{1}\left(\widehat{z}_{i}(g)-z_{i}(g)\right)+l_{2} d_{2}\left(g l(i)-z_{i}(g)\right)
$$

where, $i$ is called the index of the particle, $\varsigma$ is known as inertial coefficient, $l_{1}$ and $l_{2}$ are acceleration coefficients, $0 \leq l_{1}, l_{2} \leq 2, d_{1}, d_{2}$ are random numbers, $0 \leq d_{1}, d_{2} \leq 1$. $v_{i}(g)$ is particle's velocity at $t^{t h}$ time, $z_{i}(g)$ is the position of a particle at $t^{t h}$ time. $\left.\widehat{z}_{i}(g)\right)$ and $g l(i)$ are particle's (each individual one) best and swarm's best individual.

For each $\left(p_{i}\right) \in P$, the position is updated by the formula given by

$$
z_{i}(g+1)=z_{i}(g)+v_{i}(g+1)
$$

Interested readers may explore [19] for more details.

\section{Optimized Quantum-Inspired Self-Supervised Neural Network (Opti- QISNet) Model}

In this proposed Opti-QISNet model, the Quantum-Inspired Self-supervised Neural Network (QISNet) architecture [16, 17] is characterized by a novel quantuminspired optimized multi-level sigmoidal (Opti-QSig) activation function suitable for optimal adaptive thresholding of MR images, thereby enabling precise multi-level segmentation. The basic architecture of the Opti-QISNet mimics the QISNet architecture and the trinity layers of the Opti-QISNet architecture are arranged as input, hidden or intermediate and output comprising quantum neurons (qubits) as shown in Figure 1. The input layer of the optimized quantum-

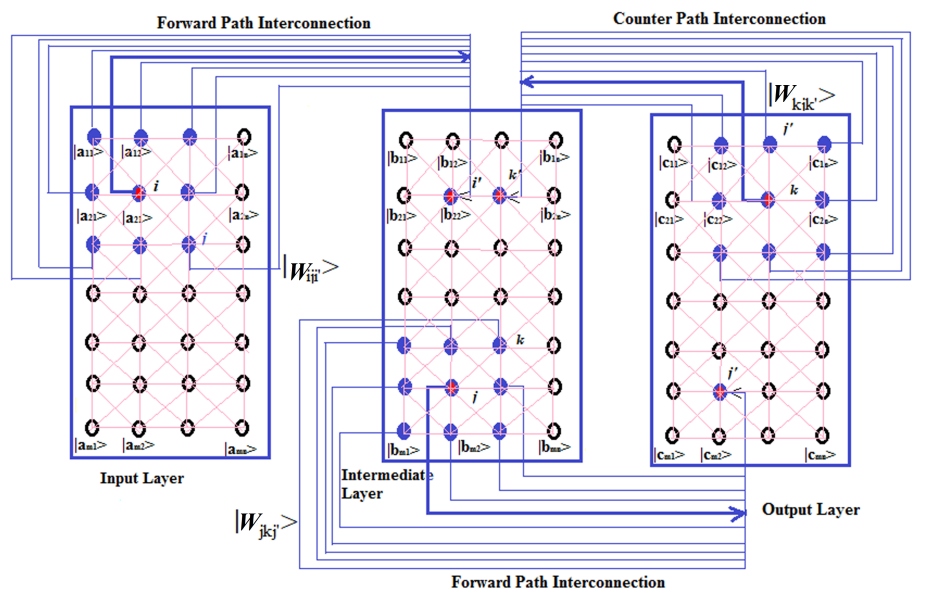

Figure 1: Quantum-Inspired Self-Supervised Neural Network (QISNet) Architecture [17] (Only one Inter-layer connection is shown between two successive layers for better visibility). 
inspired self-supervised neural network model receives normalized fuzzified image pixels in quantum formalism and propagates them forward to the successive layers (intermediate and output layer) for further processing. Each network layer is composed of quantum neurons as shown in the following image matrix.

$$
\left[\begin{array}{ccccc}
\left|\phi_{11}\right\rangle & \left|\phi_{12}\right\rangle & \left|\phi_{13}\right\rangle & \ldots & \left|\phi_{1 n}\right\rangle \\
\ldots & \ldots & \ldots & \ldots & \ldots \\
\ldots & \ldots & \ldots & \ldots & \ldots \\
\ldots & \ldots & \ldots & \ldots & \ldots \\
\left|\phi_{m 1}\right\rangle & \left|\phi_{m 2}\right\rangle & \left|\phi_{m 3}\right\rangle & \ldots & \left|\phi_{m n}\right\rangle
\end{array}\right]
$$

where, $\phi_{i j}$ corresponds a qubit or quantum state. Each layer of the Opti-QISNet model are connected through intra-connection strength $\frac{\pi}{2}$ (Quantum Logic 1). Each candidate quantum neuron cum pixel forms an 8-connected and spatially organized neighborhood neurons at each layer of the underlying architecture and inter-connected to the next subsequent layer for forward propagation of the information in quantum formalism. The output layer of Opti-QISNet is also inter-connected to intermediate layer in same 8-connected neighborhood fashion via counter-propagation of the intermediate quantum outputs.

The inter-connection weights and the activation represented as qubits in OptiQISNet model are adaptive and updated using quantum rotation gate. The angle associated with the rotation operation is determined by the relative difference measure of the candidate neuron and its neighborhood neurons. Consider the inter-linked strength between two adjacent layers of the suggested network model (as shown in Figure 1) be designated as $\mathcal{W}_{i, j, i^{\prime}}$ which is defined as

$$
\left|\mathcal{W}_{i, j, i^{\prime}}\right\rangle=\left[\begin{array}{c}
\cos \left(\frac{\pi}{2} \omega_{i, j}\right) \\
\sin \left(\frac{\pi}{2} \omega_{i, j}\right)
\end{array}\right]
$$

The angle of rotation, $\omega_{i, j}$ is determined in a such a way that it can detect the difference between the foreground and background pixels.

$$
\omega_{i, j}=1-\left(\mathcal{X}_{i}-\mathcal{X}_{i, j}\right) ; j \in\{1,2,3, \ldots 8\}
$$

where, the quantum fuzzy membership intensity grade at the $i^{\text {th }}$ candidate pixel and one of its 8-connected neighborhood $j^{\text {th }}$ pixel is $\mathcal{X}_{i}$ and $\mathcal{X}_{i, j}$, respectively. In this quantum-inspired and optimized self-supervised procedure, four distinct optimal activation schemes have been adopted and one such activation is the quantum-inspired fuzzy context sensitive activation, $\eta_{i}$ which is defined on the 8-connected neighborhood spatial intensity information as

$$
\left|\eta_{i}\right\rangle=\left[\begin{array}{c}
\cos \alpha_{i} \\
\sin \alpha_{i}
\end{array}\right]
$$

where

$$
\alpha_{i}=2 \pi \times\left(\sum_{j} \mathcal{X}_{i, j}\right)
$$


The activation enables the quantum neuron to propagate to the next layer using a bi-directional procedure followed by optimized information processing of the QISNet architecture inspired by the basic quantum neural network input-output model $[20,17]$ as

$$
\left|\phi^{l}\left(\mathcal{X}_{i}\right)\right\rangle=\sigma_{\text {opt }}\left(\sum_{j}^{m \times n} f^{(l-1)}\left(\mathcal{X}_{i}\right)\left\langle\mathcal{W}_{j}^{l} \mid \eta_{i}^{l}\right\rangle\right)
$$

where, $\left|\phi^{l}\left(\mathcal{X}_{i}\right)\right\rangle$ denotes the intermediate output of the $i^{\text {th }}$ candidate quantum neuron at the network layer in $l^{\text {th }}$ sample. $\sigma_{o p t}$ is the Quantum-inspired Optimized Multi-level Sigmoidal (Opti-QSig) activation function with activation as $\left|\eta_{i}^{l}\right\rangle$ and described in the following subsection 5.1. The output $\left|\phi^{l}\left(\mathcal{X}_{i}\right)\right\rangle$ can be written as

$$
\begin{array}{r}
\left|\phi^{l}\left(\mathcal{X}_{i}\right)\right\rangle=f\left(\frac{\pi}{2} \delta_{i}^{l}-\arg \left\{\sum_{j}^{m \times n} f^{l}\left(\omega_{j, i}\right) f^{l-1}\left(\mathcal{X}_{i}\right)-f^{l}\left(\eta_{i}\right)\right\}\right)= \\
\sigma_{\text {opt }}\left(\sum_{j}^{m \times n} f^{l-1}\left(\mathcal{X}_{i}\right)\left(\cos \left(\omega_{j, i}^{l}-\alpha_{i}^{l}\right)+\alpha \sin \left(\omega_{j, i}^{l}-\alpha_{i}^{l}\right)\right)\right)
\end{array}
$$

Here, the designated rotation angle associated with the inter-connection weights between input neuron $j$ to output neuron $i$ is $\omega_{j, i}^{l}$ and $\delta_{i}^{l}$ is the phase transfer parameter. The true classical output state $(|1\rangle)$ from $i^{\text {th }}$ quantum neuron is obtained by considering the imaginary section ( $\sin$ ) of the above expression where $\alpha$ is an imaginary unit. Assume that the inter-connection weights between the input and hidden layers of the Opti-QISNet model is denoted as $\left|\mathcal{W}_{k, j}^{l}\right\rangle$ and for the hidden layer to output layer as $\left|\mathcal{W}_{j, i}^{l}\right\rangle$ in $l^{\text {th }}$ sample sets. The activation at the hidden and output layers are designated using $\left|\eta_{j}^{l}\right\rangle$ and $\left|\eta_{i}^{l}\right\rangle$, respectively. Considering, any quantum candidate neuron $k$ from the sample of input neurons at the input layer, the corresponding seed neuron at the hidden layer be $j$ and the output candidate neuron $i$, the response at the $i^{\text {th }}$ neuron in $l^{\text {th }}$ sample sets is expressed as

$$
\begin{array}{r}
\left|\phi^{l}\left(\mathcal{X}_{i}\right)\right\rangle=\sigma_{\text {opti }}\left(\sum_{j}^{m \times n} f\left(\frac{\pi}{2} y_{j}^{l}\right)\left\langle\varphi_{j i}^{l} \mid \eta_{j}^{l}\right\rangle\right) \\
=\sigma_{\text {opti }}\left(\sum _ { j } ^ { m \times n } f \left(\frac{\pi}{2} \times \sigma_{o p t i}\left(\sum_{k}^{m \times n} f\left(\frac{\pi}{2} y_{j}^{l, d}\right)\right.\right.\right. \\
\left.\left.\left\langle\mathcal{W}_{k j}^{l} \mid \eta_{k}^{l}\right\rangle\right)\left\langle\mathcal{W}_{j i}^{l} \mid \eta_{j}^{l}\right\rangle\right)
\end{array}
$$

i.e.,

$$
\begin{array}{r}
\left|\phi^{l}\left(\mathcal{X}_{i}\right)\right\rangle= \\
\sigma_{o p t i}\left(\sum _ { j } ^ { m \times n } f \left(\frac{\pi}{2} \times \sigma_{o p t i}\left(\sum_{k}^{m \times n} f\left(\frac{\pi}{2} y_{j}^{l}\right) \cos \left(\omega_{k, j}^{l}-\alpha_{j}^{l}\right)\right.\right.\right. \\
\left.\left.\left.\cos \left(\omega_{j, i}^{l}-\alpha_{i}^{l}\right)+\alpha \sin \left(\omega_{k, j}^{l}-\alpha_{j}^{l}\right) \sin \left(\omega_{j, i}^{l}-\alpha_{i}^{l}\right)\right)\right)\right)
\end{array}
$$


Here, $\alpha$ is an imaginary number.

\subsection{Quantum-Inspired Optimized Multi-level Sigmoidal (Opti-QSig) activation function}

The suggested Opti-QISNet model is characterized using four distinct adaptive activation schemes. The Opti-QSig is optimized using quantum-inspired meta-heuristics [19] with Otsu's [18] multi-level thresholding as fitness function and is found suitable for optimal thresholding of the images with wide variation of gray-scales. The Opti-QSig activation function, $f_{O p t i-Q S i g}$ is defined as

$$
f_{O p t i-Q \operatorname{Sig}}(\mathcal{X})=\frac{1}{\zeta_{\theta}+e^{-\mu(\mathcal{X}-\eta)}}
$$

where, $\lambda$ and $\eta$ represent steepness or slope of the function with steepness factor $\mu$ and activation in quantum formalism, respectively. In addition, $\zeta_{\theta}$ designates the class responses over 8-connected neighborhood based pixels and is defined as

$$
\zeta_{\theta}=\frac{\Omega_{N}}{\lambda_{\theta}-\lambda_{\theta-1}}
$$

where, $\lambda_{\theta}$ and $\lambda_{\theta-1}$ are the responses of two adjacent class levels $\theta$ and $\theta-1$, respectively. $\Omega_{N}$ is the maximum contribution of 8-connected neighborhood pixels. Suitable advocating of $\lambda_{\theta}$ modifies the Opti-QSig activation function as given in Eq 19 to the generalized Opti-QSig activation function. Combination of different subnormal responses $f_{\mu_{\lambda_{\theta}}}$ results in the multi-class response of the generalized Opti-QSig activation function in quantum formalism given as

$$
f_{O p t i-Q S i g}\left(\mathcal{X} ; \zeta_{\theta}, \lambda_{\theta}\right)=\frac{1}{\zeta_{\theta}+e^{-\mu\left(\mathcal{X}-(\theta-1) \lambda_{\theta-1}-\eta\right)}}, 0 \leq f_{\mu_{\lambda_{\theta}}} \leq \frac{\pi}{2}
$$

The resultant form of Opti-QSig activation function can be written as

$$
\begin{array}{r}
f_{R}(\mathcal{X})=\sum_{\theta=1}^{\mathcal{L}} f_{\text {Opti-QSig }}\left(\mathcal{X}+(\theta-1) \lambda_{\theta-1}\right) \\
\forall(\theta-1) \lambda_{\theta-1} \leq \mathcal{X} \leq \theta \lambda_{\theta}
\end{array}
$$

Modification can be done on Eq 22 substituting Eq 21 as

$$
f_{R}\left(\mathcal{X} ; \zeta_{\theta}, \lambda_{\theta}\right)=\sum_{\theta=1}^{\mathcal{L}} \frac{1}{\zeta_{\theta}+e^{-\mu\left(x-(\theta-1) \lambda_{\theta-1}-\eta\right)}}
$$

The Opti-QSig activation functions with various slopes and class levels are shown in Fig 2. However, the activation parameter $\eta$ used in the Opti-QSig activation function is appropriate for uniformly distribution of intensity and hence, gray-level segmentation accuracy degrades for MR images due to wide variations of gray-scales having heterogeneous response exhibited over the 8connected region. In order to tackle this problem, we have entrusted on adaptive 


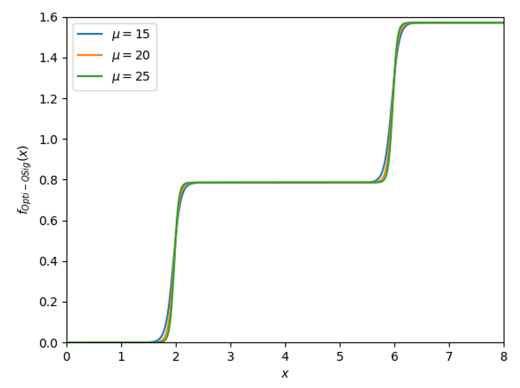

(a) $\mathcal{L}=3$

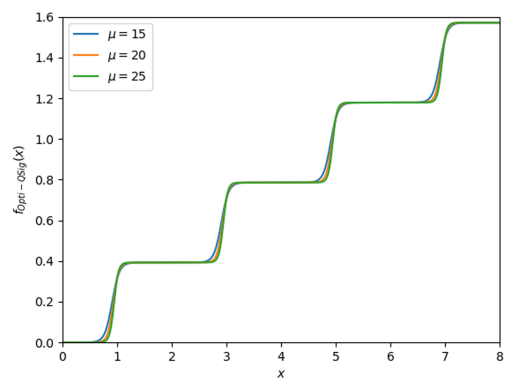

(c) $\mathcal{L}=5$

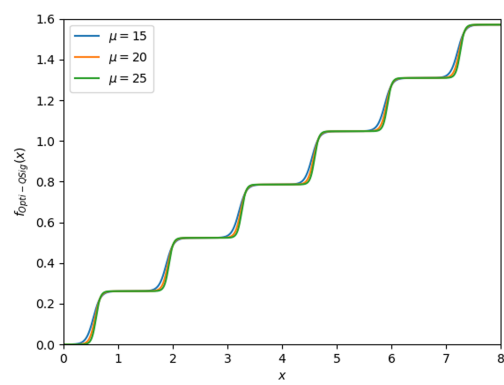

(e) $\mathcal{L}=7$

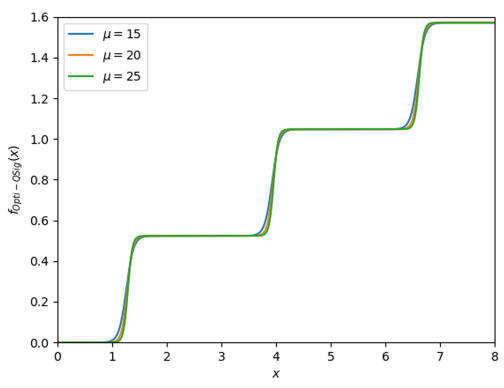

(b) $\mathcal{L}=4$

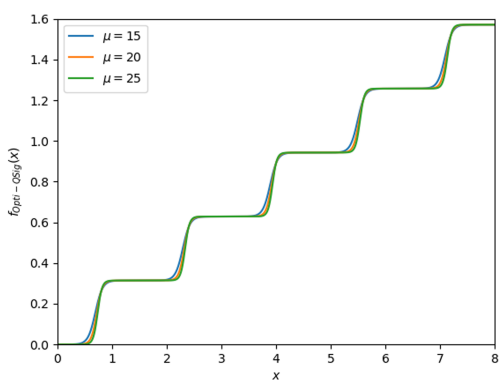

(d) $\mathcal{L}=6$

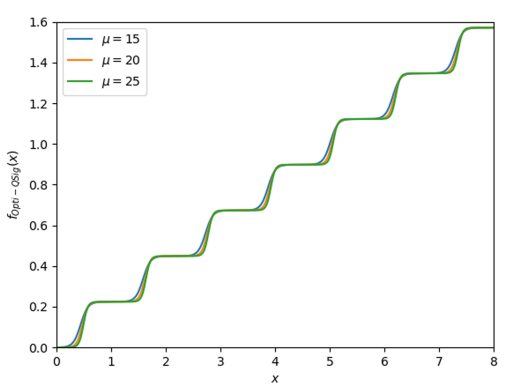

(f) $\mathcal{L}=8$

Figure 2: Multi-level class outcome of Opti-QSig activation function for $\mu=15,20,25$ and distinct classes $(\mathrm{a}) \mathcal{L}=3,(\mathrm{~b}) \mathcal{L}=4,(\mathrm{c}) \mathcal{L}=5,(\mathrm{c}) \mathcal{L}=6,(\mathrm{c}) \mathcal{L}=7,(\mathrm{~d}) \mathcal{L}=8$. 
and optimal thresholding schemes using trinity quantum-inspired meta-heuristic approaches [19] with Otsu's [18] multi-level thresholding as fitness function. In this paper, Dynamic Susceptibility Contrast (DSC) brain MR image are processed through the different layers of quantum neurons in terms of fuzzy membership grades of the pixels in quantum formalism. There are four distinct adaptive activation schemes used for the activation parameter $\eta$ in the proposed Opti-QSig activation function as provided below [50, 51, 52].

(1) Activation based on $\beta$-distributed intensity of 8-connected neighborhood image pixels $\left(\eta_{\beta}\right)$.

(2) Activation based on skewness $\left(\eta_{\chi}\right)$.

(3) Activation based on fuzzy graded pixel heterogeneous intensity of 8-connected neighborhood $\left(\eta_{\xi}\right)$.

(4) Activation based on fuzzy cardinality estimation of 8-connected neighborhood $\left(\eta_{\nu}\right)$.

The optimized multi-class level, $\left(\mathcal{L}_{\theta}\right)$ for fixed number of boundaries or class $\mathcal{L}$ is defined in a closed set $\mathcal{F}_{\lambda_{\omega}}$ as

$$
\mathcal{F}_{\lambda_{\theta} \mathcal{L}}=\left\{\left\{\lambda_{\theta \mathcal{L}}\right\}, \mathcal{L}=4,5,6,7,8\right\}
$$

In order to obtain a number of optimal thresholds $\left\{\theta_{1}, \theta_{2}, \cdots, \theta_{C_{l}-1}\right\}$, Otsu's multi-level image thresholding [18] is incorporated to maximizes the spread of the classes, and is defined as [18]

$$
\mathcal{O}=f_{n}\left\{\theta_{1}, \theta_{2}, \cdots, \theta_{C_{l}-1}\right\}=\sum_{k=1}^{C_{l}} \omega_{k}\left(\rho_{k}-\rho\right)
$$

where, $C_{l}$ represents the number of defined classes in $\mathcal{C}=\left\{C_{1}, C_{2}, \ldots, C_{C_{l}}\right\}$ and

$$
\omega_{k}=\sum_{i \in C_{k}} p_{i}, \rho_{k}=\sum_{i \in C_{k}} i p_{i} / \omega_{k}
$$

where, $p_{i}$ designates the the $i^{\text {th }}$ pixel and $\omega_{k}$ represents the probability of class $C_{k}$ with the mean value given by $\mu_{k}$. The mean of the class $\mathcal{C}$ is given by $\mu$. In this work, for each multi-class level $\mathcal{L}=\{4,6,8\}$ four sets $\mathcal{F}_{\lambda_{\theta \mathcal{L}}}=\left\{\lambda_{1}, \lambda_{2}, \lambda_{3}, \lambda_{4}\right\}$ of class boundary are computed using Otsu's method [18] as fitness values in quantum formalism and are optimized by trinity of quantum-inspired metaheuristics (QDE, QACO and QPSO). One set of such example is for slice \#3 using QDE as given below.

$$
\begin{gathered}
\mathcal{F}_{\lambda_{\omega(4)}}=\frac{\pi}{2} \times\{\{0,0.23,0.38,0.89,1\},\{0,0.25,0.42,0.98,1\} \\
\{0,0.25,0.34,0.64,1\},\{0,0.24,0.38,0.66,1\}\} \\
\mathcal{F}_{\lambda_{\omega(6)}}=\frac{\pi}{2} \times\left\{\begin{array}{c}
\{0,0.12,0.19,0.29,0.35,0.94,1\},\{0,0.11,0.21,0.28,0.44,0.76,1\} \\
\{0,0.22,0.27,0.45,0.61,0.87,1\},\{0,0.19,0.27,0.48,0.50, .96,1\}\}
\end{array}\right.
\end{gathered}
$$




$$
\begin{array}{r}
\mathcal{F}_{\lambda_{\omega(8)}=\frac{\pi}{2} \times\{}\{0,0.04,0.09,0.16,0.31,0.46,0.50,0.62,1\}, \\
\{0,0.03,0.12,0.17,0.20,0.31,0.43,0.82,1\}, \\
\{0,0.12,0.17,0.22,0.46,0.53,0.58,0.72,1\}, \\
\{0,0.06,0.16,0.31,0.46,0.61,0.76,0.96,1\}\}
\end{array}
$$

\section{Inter-connection Weight Adjustment and Convergence Analysis of the Opti-QISNet Model}

The inter-linked weights are advocated using the optimal threshold offered by Otsu's multi-class thresholding, thereby enabling the proposed Opti-QISNet model to converge. It has significant relevance in a self-supervised network models to obtain optimal segmented images. The segmented MR images are obtained once the network stabilizes otherwise it undergoes further processing in the intermediate layer. Quantum rotation gates are employed to update the inter-linked weights and the activation between the layers of the network model as

$$
\begin{aligned}
\left|\mathcal{W}^{l+1}\right\rangle & =\left(\begin{array}{cc}
\cos \triangle \omega & -\sin \triangle \omega \\
\sin \triangle \omega & \cos \triangle \omega
\end{array}\right)\left|\mathcal{W}^{l}\right\rangle \\
\left|\eta^{l+1}\right\rangle & =\left(\begin{array}{cc}
\cos \triangle \alpha & -\sin \triangle \alpha \\
\sin \triangle \alpha & \cos \triangle \alpha
\end{array}\right)\left|\eta^{l}\right\rangle
\end{aligned}
$$

where,

$$
\omega^{l+1}=\omega^{l}+\triangle \omega^{l}
$$

and

$$
\alpha^{l+1}=\alpha^{l}+\triangle \alpha^{l}
$$

where, the change in phase angles $\triangle \omega^{l}$ and $\triangle \alpha^{l}$ are measured using Equations 32 and 33, respectively. In this Opti-QISNet, the loss function relies on the interconnection strengths and is calculated as

$$
\mathcal{E}(\omega, \alpha)=\frac{1}{N} \sum_{i=1}^{N} \sum_{j=1}^{8}\left[\mathcal{W}_{i j}\left(\omega_{i j}, \alpha_{i}\right)^{l+1}-\mathcal{W}_{i j}\left(\omega_{i j}, \alpha_{i}\right)^{l}\right]^{2}
$$

Hence, the inter-connected strengths at epoch $l$ is $\mathcal{W}_{i j}\left(\omega_{i j}, \alpha_{i}\right)^{l} . \mathcal{E}(\omega, \alpha)$ is designated as the loss function over phase angles $\omega$ and $\alpha$. Consider

$$
\begin{aligned}
& \mathcal{V}^{l}=\omega^{l}-\bar{\omega} \\
& \mathcal{M}^{l}=\alpha^{l}-\bar{\alpha}
\end{aligned}
$$

and

$$
\begin{gathered}
\mathcal{D}^{l}=\omega^{l+1}-\omega^{l}=\mathcal{V}^{l+1}-\mathcal{V}^{l} \\
\mathcal{P}^{l}=\alpha^{l+1}-\alpha^{l}=\mathcal{M}^{l+1}-\mathcal{M}^{l}
\end{gathered}
$$


where, $\bar{\omega}$ and $\bar{\alpha}$ are the optimal phase angles for inter-connection weights and activation respectively. The loss function $\mathcal{E}(\omega, \alpha)$ is differentiated with respect to $\omega, \alpha$ as

$$
\begin{array}{r}
\frac{\partial \mathcal{E}(\omega, \alpha)}{\partial \omega_{i j}}= \\
\frac{2}{N} \sum_{i=1}^{N} \sum_{j=1}^{8} \Delta \mathcal{W}_{i j}\left(\omega_{i k}, \alpha_{i j}\right)^{l}\left[\frac{\partial \mathcal{W}_{i j}\left(\omega_{i j}, \alpha_{i j}\right)^{l+1}}{\partial \omega_{i j}}-\frac{\partial \mathcal{W}_{i j}\left(\omega_{i j}, \alpha_{i j}\right)^{l}}{\partial \omega_{i j}}\right] \\
\frac{\partial \mathcal{E}(\omega, \alpha)}{\partial \alpha_{i}}= \\
\frac{2}{N} \sum_{i=1}^{N} \Delta \mathcal{W}_{i}\left(\omega_{i}, \alpha_{i}\right)^{l}\left[\frac{\partial \mathcal{W}_{i}\left(\omega_{i}, \alpha_{i}\right)^{l+1}}{\partial \alpha_{i}}-\frac{\partial \mathcal{W}_{i}\left(\omega_{i}, \alpha_{i}\right)^{l}}{\partial \alpha_{i}}\right]
\end{array}
$$

where

$$
\Delta \mathcal{W}_{i j}\left(\omega_{i}, \alpha_{i j}\right)^{l}=\left|\mathcal{W}_{i j}\left(\omega_{i j}, \alpha_{i}\right)^{l+1}-\mathcal{W}_{i j}\left(\omega_{i j}, \alpha_{i}\right)^{l}\right|
$$

The following equations evaluate the change in phase or angles $(\Delta \omega$ and $\triangle \alpha)$ of the rotation gate as

$$
\begin{gathered}
\triangle \omega_{i j}^{l}=-\rho_{i j}\left\{\frac{\partial \mathcal{E}(\omega, \alpha)^{l}}{\partial \omega_{i j}^{l}} \mathcal{E}(\omega, \alpha)^{l}\right\}^{\frac{1}{\iota}} \\
\triangle \alpha_{i}^{l}=-\kappa_{i}\left\{\frac{\partial \mathcal{E}(\omega, \alpha)}{\partial \alpha_{i}^{l}} \mathcal{E}(\omega, \alpha)^{l}\right\}^{\frac{1}{\iota}}
\end{gathered}
$$

where, $\rho_{i j}$ and $\kappa_{i}$ refer to the learning rate of quantum-inspired self-supervised optimized learning and are evaluated as

$$
\begin{array}{r}
\rho_{i j}=\mathcal{X}_{i}-\mathcal{X}_{i j} \forall j=1,2 \ldots 8 \\
a n d \kappa_{i}=\left(\sum_{j} \mathcal{X}_{i, j}\right) \forall j=1,2 \ldots 8
\end{array}
$$

The sequences of $\left\{\omega^{l}\right\}$ and $\left\{\alpha^{l}\right\}$ converge super-linearly subject to the following conditions [53].

$$
\lim _{l \rightarrow \infty} \frac{\left\|\omega^{l+1}-\bar{\omega}\right\|}{\left\|\omega^{l}-\bar{\omega}\right\|} \leq 1
$$

and

$$
\left\|\mathcal{V}^{l+1}\right\|=O\left\|\mathcal{D}^{l}\right\|
$$

Also,

$$
\lim _{l \rightarrow \infty} \frac{\left\|\alpha^{l+1}-\bar{\alpha}\right\|}{\left\|\alpha^{l}-\bar{\alpha}\right\|} \leq 1
$$

and

$$
\left\|\mathcal{M}^{l+1}\right\|=O\left\|\mathcal{P}^{l}\right\|
$$


According to $L$-Lipschitz continuity, the convergence of the sequences $\left\{\omega^{l}\right\}$ can be shown as [54]

$$
\begin{array}{r}
\mathcal{E}\left(\omega^{l+1}\right) \leq \mathcal{E}\left(\omega^{l}\right)+\left\langle\nabla_{\omega} \mathcal{E}\left(\omega^{l}\right), \omega^{l+1}-\omega^{l}\right\rangle+\frac{L}{2}\left\|\omega^{l+1}-\omega^{l}\right\|^{2} \\
=\mathcal{E}\left(\omega^{l}\right)+\left\langle\nabla_{\omega} \mathcal{E}\left(\omega^{l}\right)-\rho \nabla_{\omega} \mathcal{E}\left(\omega^{l}\right)\right\rangle+\frac{L}{2}\left\|-\rho \nabla_{\omega} \mathcal{E}\left(\omega^{l}\right)\right\|^{2} \\
=\mathcal{E}\left(\omega^{l}\right)-\rho\left\|\nabla_{\omega} \mathcal{E}\left(\omega^{l}\right)\right\|^{2}+\rho^{2} \frac{L}{2}\left\|\nabla_{\omega} \mathcal{E}\left(\omega^{l}\right)\right\|^{2} \\
=\mathcal{E}\left(\omega^{l}\right)-\rho\left(1-\rho \frac{L}{2}\right)\left\|\nabla_{\omega} \mathcal{E}\left(\omega^{l}\right)\right\|^{2} \\
\leq \mathcal{E}\left(\omega^{l}\right)-\frac{\rho}{2}\left\|\nabla_{\omega} \mathcal{E}\left(\omega^{l}\right)\right\|^{2}\left(A s s u m i n g, \rho \in\left(0, \frac{1}{L}\right]\right) \\
\leq \mathcal{E}(\bar{\omega})+\left\langle\nabla_{\omega} \mathcal{E}\left(\omega^{l}\right), \omega^{l}-\bar{\omega}\right\rangle-\frac{\rho}{2}\left\|\nabla_{\omega} \mathcal{E}\left(\omega^{l}\right)\right\|^{2},(\mathcal{E} \text { is convex }) \\
=\mathcal{E}(\bar{\omega})+\frac{1}{2 \rho}\left(\left\|\omega^{l}-\bar{\omega}\right\|^{2}-\left(\left\|\omega^{l}\right\|^{2}-2\left\langle\omega^{l}, \bar{\omega}\right\rangle+\|\bar{\omega}\|^{2}-2 \rho\left\langle\nabla_{\omega} \mathcal{E}\left(\omega^{l}\right), \omega^{l}-\bar{\omega}\right\rangle+\rho^{2}\left\|\nabla_{\omega} \mathcal{E}\left(\omega^{l}\right)\right\|^{2}\right)\right) \\
=\mathcal{E}(\bar{\omega})+\frac{1}{2 \rho}\left(\left\|\omega^{l}\right\|-\bar{\omega} \|^{2}-\left(\left\|\omega^{l}-\rho \nabla_{\omega} \mathcal{E}\left(\omega^{l}\right)\right\|^{2}-2\left\langle\omega^{l}-\nabla_{\omega} \mathcal{E}\left(\omega^{l}\right), \bar{\omega}\right\rangle+\|\bar{\omega}\|^{2}\right)\right) \\
=\mathcal{E}(\bar{\omega})+\frac{1}{2 \rho}\left(\left\|\omega^{l}-\bar{\omega}\right\|^{2}-\left\|\omega^{l+1}-\bar{\omega}\right\|^{2}\right) \\
\therefore, \mathcal{E}\left(\omega^{l+1}\right)-\mathcal{E}(\bar{\omega}) \leq \frac{1}{2 \rho}\left(\left\|\omega^{l}-\bar{\omega}\right\|^{2}-\left\|\omega^{l+1}-\bar{\omega}\right\|^{2}\right)
\end{array}
$$

Similarly, it can also be shown that

$$
\mathcal{E}\left(\alpha^{l+1}\right)-\mathcal{E}(\bar{\alpha}) \leq \frac{1}{2 \rho}\left(\left\|\alpha^{l}-\bar{\alpha}\right\|^{2}-\left\|\alpha^{l+1}-\bar{\alpha}\right\|^{2}\right)
$$

Now, according to Thaler formula

$$
\begin{aligned}
& \mathcal{E}\left(\omega^{l+1}, \alpha^{l+1}\right)-\mathcal{E}\left(\omega^{l}, \alpha^{l}\right)= \\
& {\left[\begin{array}{ll}
\triangle \omega_{i j}^{l} & \triangle \alpha_{i}^{l}
\end{array}\right]\left[\begin{array}{c}
\frac{\partial \mathcal{E}(\omega, \alpha)^{l}}{\partial \omega_{i j}^{l}} \\
\frac{\partial \mathcal{E}(\omega, \alpha)^{l}}{\partial \alpha_{i j}^{l}}
\end{array}\right]+O\left[\left\|\triangle \omega_{i j}^{l} \quad \Delta \alpha_{i}^{l}\right\|\right]} \\
& \approx\left[\left\{-\rho_{i j} \frac{\partial \mathcal{E}(\omega, \alpha)^{l}}{\partial \omega_{i j}^{l}}\right\}^{2}+\left\{-\rho_{i} \frac{\partial \mathcal{E}(\omega, \alpha)^{l}}{\partial \alpha_{i j}^{l}}\right\}^{2}\right]\left\{\mathcal{E}\left(\omega^{l}, \alpha^{l}\right)\right\}^{\frac{1}{l}}
\end{aligned}
$$

It is obvious that $\left(\mathcal{E}\left(\omega^{l+1}, \alpha^{l+1}\right)-\mathcal{E}\left(\omega^{l}, \alpha^{l}\right)\right) \leq 0$ and the sequences of $\left\{\omega^{l}\right\}$ and $\left\{\alpha^{l}\right\}$ are monotonically decreasing as

$$
\lim _{l \rightarrow \infty} \mathcal{E}\left(\omega^{l}, \alpha^{l}\right)=(\bar{\omega}, \bar{\alpha})
$$


and

$$
\lim _{l \rightarrow \infty} \frac{\left\|\mathcal{E}\left(\omega^{l+1}, \alpha^{l+1}\right)-(\bar{\omega}, \bar{\alpha})\right\|}{\left\|\mathcal{E}\left(\omega^{l}, \alpha^{l}\right)-(\bar{\omega}, \bar{\alpha})\right\|} \leq 1
$$

Convergence analysis is also demonstrated experimentally for the trinity quantuminspired meta-heuristics with four distinct activation $\left(\eta_{\beta}, \eta_{\chi}, \eta_{\xi}, \eta_{\nu}\right)$ as shown in Figure 3.

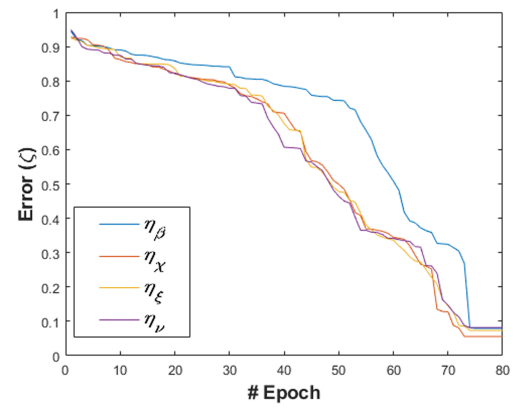

(a) QDE-QISNet

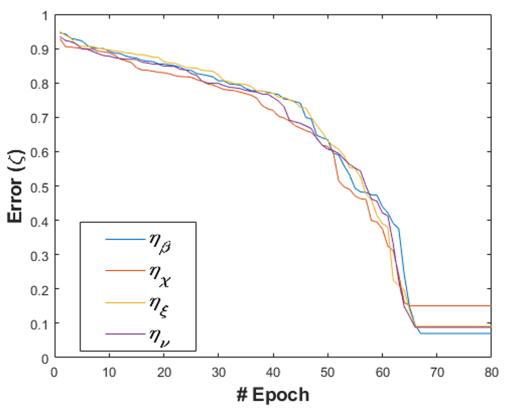

(c) QPSO-QISNet

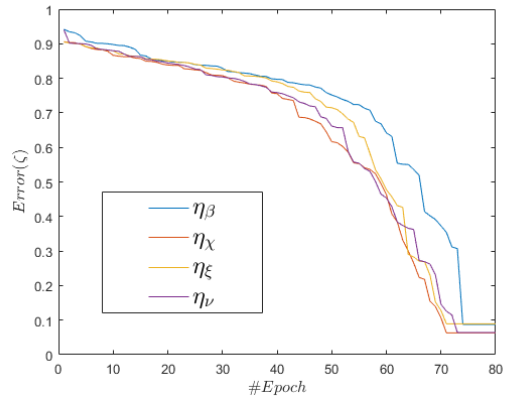

(b) QACO-QISNet

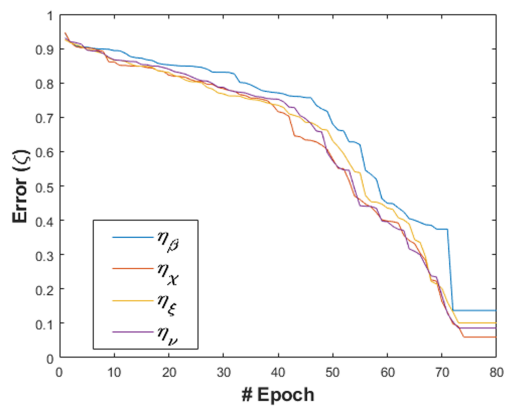

(d) QIS-Net

Figure 3: Convergence Graph using four different activation for the proposed (a) QDE-QISNet (b) QACO-QISNet (c) QPSO-QISNet and (d) QIS-Net

An integrated and optimized quantum-inspired self-supervised framework using QISNet model for fully automated MR images segmentation is provided in Figure 4.

\section{Results and Discussion}

\subsection{Data Set}

Rigorous experiments have been conducted on Dynamic Susceptibility Contrast (DSC) Brain Magnetic Resonance images which are available from the Nature Data repository [55]. In contrast to the automatic brain lesion segmentation, experiments have been performed using the proposed Opti-QISNet, 


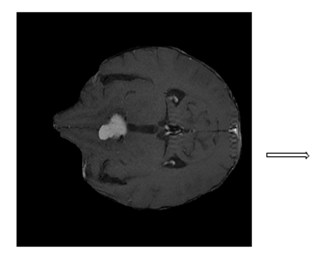

Input skull-tripped $\mathrm{T}_{1}$-weighted CE Image

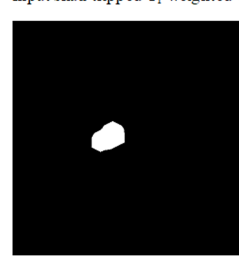

Final segmented tumour region obtained after masking

operation

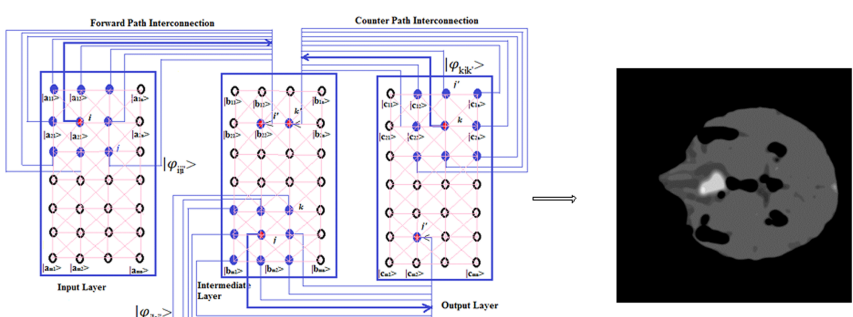

Opti-QISNet Model



Figure 4: Proposed quantum-inspired self-supervised and optimized framework for optimal segmentation of Brain MR images

Quantum-inspired Bi-directional Self-organizing Neural Network (QIBDS Net) [16], Quantum-Inspired Self-supervised Neural Network (QIS-Net) model [17], OptiQIBDS Net [20], U-Net [28], FCNN [56] architectures and fuzzy-C-means clustering (FCM) [2]. The U-Net [28] and FCNN [56] architectures are trained with 2000 MR images, validated and tested on 120 and 880 contrast-enhanced DSC MR images, respectively.

\subsection{Evaluation Criteria}

A suitable evaluation scheme has been adopted using four matrices viz., Positive Predictive Value $(P P V)$, Sensitivity $(S S)$, Accuracy $(A C C)$ and Dice Similarity Score $(D S)$ [57]. The dice similarity score is often used to measure the similarity of the segmented brain lesions and regions of interest (ROIs). The accuracy measure matrices are formulated as

$$
\begin{gathered}
P P V=\frac{T_{R P}}{T_{R P}+F_{L P}} \\
S S=\frac{T_{R P}}{T_{R P}+F_{L N}} \\
A C C=\frac{T_{R P}+T_{R N}}{T_{R P}+F_{L P}+T_{R N}+F_{L N}} \\
D S=\frac{2 T_{R P}}{2 T_{R P}+F_{L P}+F_{L N}}
\end{gathered}
$$

where, $T_{R P}, F_{L P}, T_{R N}$, and $F_{L N}$ are True Positive, False Positive, True Negative and False Negative, respectively. 
Table 1: Parameter specification for QACO, QDE and QPSO

\begin{tabular}{|c|c|c|}
\hline QACO & QDE & QPSO \\
\hline Pop size: $\mathcal{R}=50$ & Pop size: $\mathcal{R}=50$ & Pop size: $\mathcal{R}=50$ \\
\hline $\begin{array}{l}\text { No. of gen: } M x G e n= \\
1000\end{array}$ & en: $M x G e n=$ & $\begin{array}{l}\text { No. of gen: } M x G e n= \\
1000\end{array}$ \\
\hline $\begin{array}{l}\text { Priori defined no.: } q_{0}= \\
0.5\end{array}$ & Scaling factor: $F=0.5$ & Inertia weight: $\omega=0.4$ \\
\hline $\begin{array}{l}\text { Persistence of trials: } t= \\
0.2\end{array}$ & $\begin{array}{l}\text { Crossover prob.: } P_{c}= \\
0.9\end{array}$ & $\begin{array}{l}\text { Accel. coeff.: } c_{1}, c_{2}= \\
0.5\end{array}$ \\
\hline $\begin{array}{l}\text { No. of thresholds: } K= \\
2-5\end{array}$ & $\begin{array}{l}\text { No. of thresholds: } \\
K=2-5\end{array}$ & $\begin{array}{l}\text { No. of thresholds: } \\
K=2-5\end{array}$ \\
\hline
\end{tabular}

\subsection{Experimental Setup}

In this current work, extensive experiments have been carried out on 3000 Dynamic Susceptibility Contrast (DSC) brain MR images of size $512 \times 512$ in a PARAM SHAVAK DL GPU System with high performance facilities provided by CDAC, India with MATLAB 2019 and Python 3.6. The sample input Dynamic Susceptibility Contrast (DSC) MR images of size $512 \times 512$ with their ground truth are shown in Fig 5. The 2D segmented images are processed though a 2D binary mask to obtain the brain lesion in the suggested Opti-QISNet framework. The lesion or brain tumor detection mask is binarized using a threshold of 0.5. Experiments are also performed on two recently developed CNN architectures suitable for medical image segmentation viz., convolutional U-Net [28] and Fully Convolutional Neural Networks [56]. The U-Net and FCNNs networks are rigorously trained using the stochastic gradient descent with learning rate of 0.02 and 0.01, respectively and batch size of 32 for U-Net allowing maximum 50 epochs to converge. The segmented output images resemble in size with the dimensions of the binary mask and the outcome 1 is considered as tumor region and 0 as background in detecting complete tumour. The pixel by pixel comparison with the manually segmented regions of interest or lesion mask allows to evaluate the dice similarity which is considered as a standard evaluation procedure in automatic medical image segmentation. The evaluation process involves the manually segmented lesion mask as ground truth and each 2D pixel is predicted as either True Positive $\left(T_{R P}\right)$ or True Negative $\left(T_{R N}\right)$ or False Positive $\left(T_{R N}\right)$ or False Negative $\left(F_{L N}\right)$.

Dey et al. have developed trinity of quantum-inspired meta-heuristics, namely QACO, QDE and QPSO in recent years [19]. The authors have employed the following set of parameters for experimental purpose, as presented in Table 1. The suggested quantum-inspired fully self-supervised optimized learning network model is experimented with the multi-level gray scale images using with distinct classes $\mathcal{L}=4,5,6,7$ and 8 , characterized by multi-class quantum-inspired sig- 
moidal activation function optimized by three quantum inspired meta-heuristics (QDE, QACO and QPSO). In this experiment, the steepness in the Opti-QSig activation, $\mu$, is varied in the range of 0.23 to 0.24 with step size of 0.001 . It has been observed that in majority cases, $\mu=0.239$ yields optimal performance. An extensive post processing on the segmented MR images is performed to remove the erroneous tumors with tiny radius which are chosen judiciously after several trials. It is seen that with a radius of 5 pixels, the segmented ROIs perform optimally while compared to the human expert segmented images since the false positive is reduced. The U-Net model [28] is experimented with various size of kernels $2 \times 2,4 \times 4$ and $6 \times 6$ with stride size 2 and it has been observed that the optimal segmentation is obtained for kernel size $=2 \times 2$ as demonstrated in Figure 6.

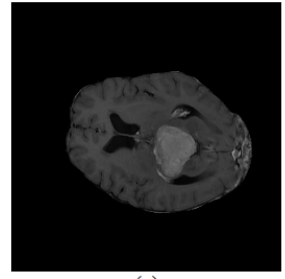

(a)

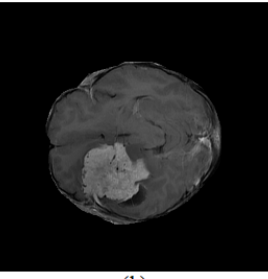

(b)

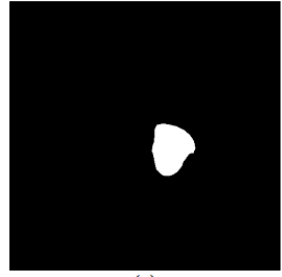

(c)

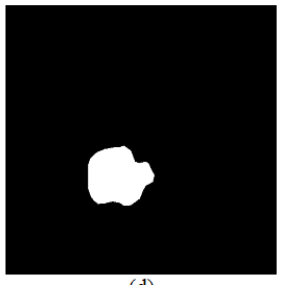

(d)

Figure 5: Skull tripped Input Brain MR image: (a) slice \#21 (b) slice no: \#3 (c) Annotated complete tumor for slice \#21 and $(d)$ for slice \#3 [55]

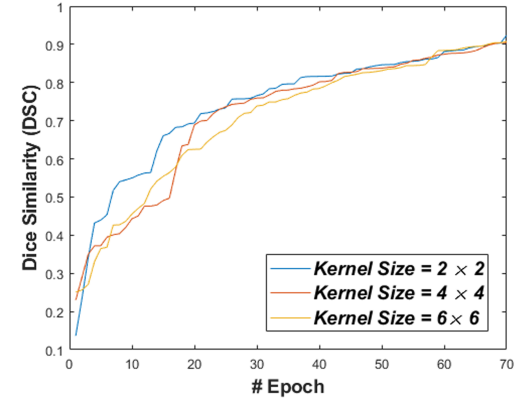

(a) U-Net

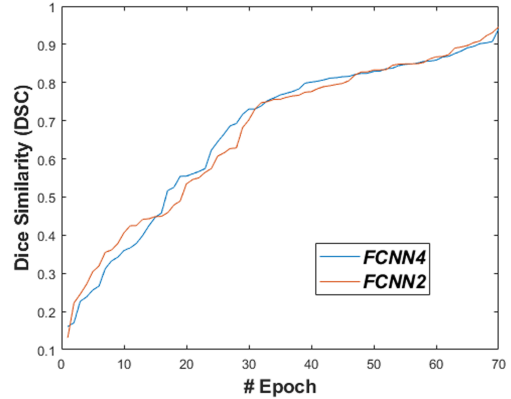

(b) FCNNs

Figure 6: Average Dice Similarity (DS) reported using (a) U-Net [28] for various kernel size and (b) Fully Convolutional Neural Networks (FCNNs) [56] during Training for 70 epochs

\subsection{Experimental Results}

Extensive experiments have been performed in the current setup and experimental outcomes are reported with the demonstration of numerical and statistical analyses using the proposed quantum-inspired self-supervised and optimized 


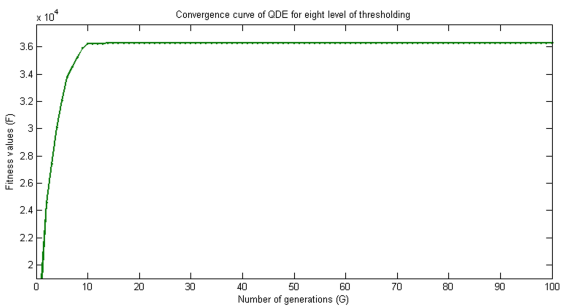

(a) QDE



(b) QACO

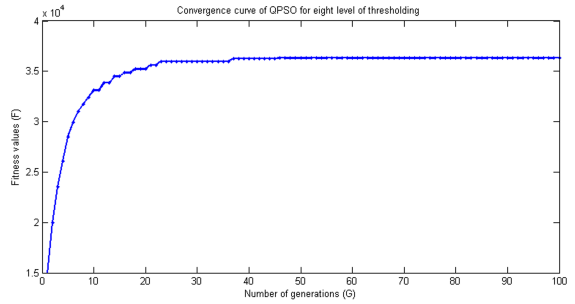

(c) QPSO

Figure 7: Average fitness reported using various quantum-inspired meta-heuristics (a) QDE (b) QACO and (c) QPSO on MR test images for multi-level $(\mathcal{L}=8)$ optimal thresholding

network model (Opti-QISNet), Quantum-inspired Bi-directional self-supervised Neural Network (QIBDS Net) [16], Quantum-Inspired Self-supervised Neural Network (QIS-Net) model [17], Opti-QIBDS Net [20], deeply supervised UNet [28], FCNNs [56] and unsupervised fuzzy-C-means clustering (FCM) [2]. The human expert segmented skull-tripped Dynamic Susceptibility Contrast (DSC) brain MR input image slices of size $512 \times 512$ ROIs are provided in Figure 5 as samples. The demonstration of Opti-QISNet segmented images followed by essential post-processed outcome on the slice no. 3 for class level $\mathcal{L}=4,6,8$ with four distinct activation schemes $\left(\eta_{\beta}, \eta_{\chi}, \eta_{\xi}, \eta_{\nu}\right)$ and optimized by quantum-inspired differential evolution (QDE) are shown in Figs 8-10. It is worth noting from the results reported in Table 2, that the suggested OptiQISNet model attains optimal outcomes for $\eta_{\xi}$ activation and with the class level $\mathcal{L}=8$. The suggested quantum-inspired optimized self-supervised operations yields optimal segmentation of brain tumors using $\mathcal{L}=8$ class with activation schemes $\eta_{\beta}, \eta_{\chi}, \eta_{\xi}$ and $\eta_{\nu}$ and are demonstrated in Figures 11-14 for QDE-QISNet, QACO-QISNet, QPSO-QISNet and QIS-Net, respectively. The segmented ROIs describing the complete tumor region after the masking procedure using fuzzy C-means clustering, U-Net and FCNNs are also reported in Figure 15.

Table 3 presents the numerical results obtained using the proposed OptiQISNet using all three quantum-inspired meta-heuristics $Q D E, Q A C O$ and $Q P S O$ and QIBDS Net [16], QISNet [17] and Opti-QIBDS Net [20] on evaluating the average accuracy $(A C C)$, dice similarity score $(D S)$, positive prediction 


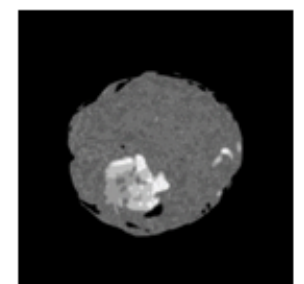

(a)

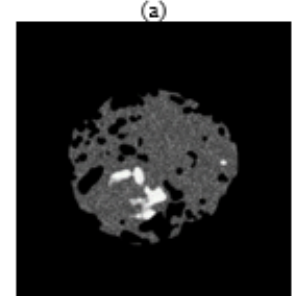

(a)



(a)

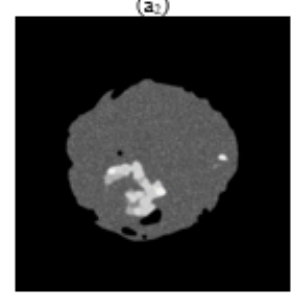

(a)

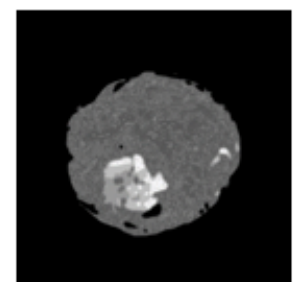

(b)



(b)

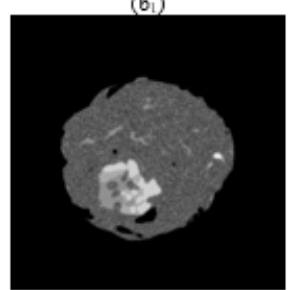

(b)

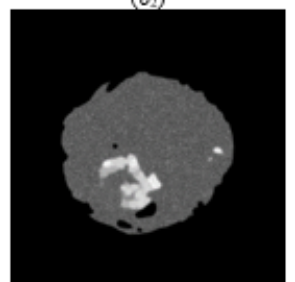

(bis)

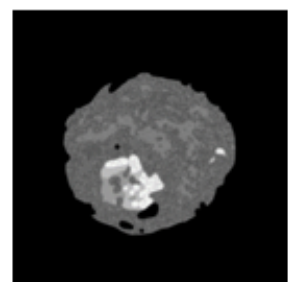

(c)

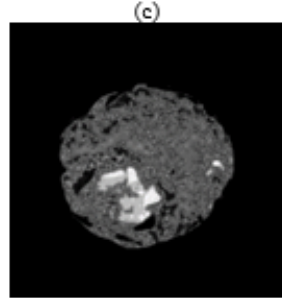

(c)



(c)

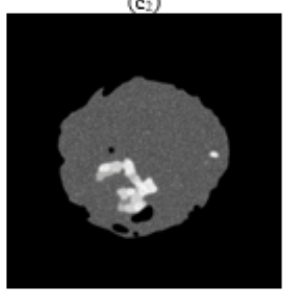

(c)

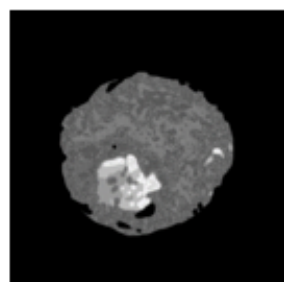

(d)

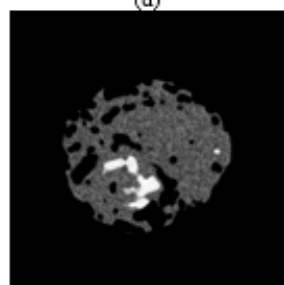

(d)

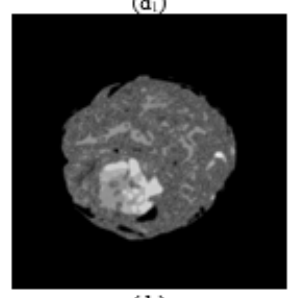

(d)

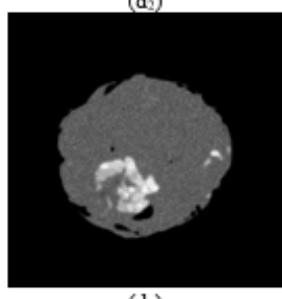

(d)

Figure 8: Segmentation using QDE-QISNet for slice \#3 with $\mathcal{L}=4$ (Activation $\left(a-a_{3}\right) \eta_{\beta},\left(b-b_{3}\right) \eta_{\chi},\left(c-c_{3}\right) \eta_{\xi},\left(d-d_{3}\right) \eta_{\nu}$. Level set $(a-d) \lambda_{1},\left(a_{1}-d_{1}\right) \lambda_{2}$, $\left.\left(a_{2}-d_{2}\right) \lambda_{3},\left(a_{3}-d_{3}\right) \lambda_{4}\right)$ 
value $(P P V)$ and sensitivity $(S S)$ as reported under $\mathcal{L}=8$ class transition levels $\left(\lambda_{1}, \lambda_{2}, \lambda_{3}, \lambda_{4}\right)$ with four distinct thresholding schemes $\left(\eta_{\beta}, \eta_{\chi}, \eta_{\xi}\right.$ and $\left.\eta_{\nu}\right)$. In addition, Table 3 summarises the results obtained using unsupervised fuzzyC-means clustering (FCM) [2], convolutional U-Net [28] and FCNN-4, FCNN2 [56] architectures for two distinct convolutional masks with size $2 \times 2,4 \times 5$ and $6 \times 6$ with stride sizes of 2 . However, the convolutional based architectures (U-Net and FCNNs) marginally outperform our proposed optimized version of Quantum-Inspired Self-supervised Neural Network model (Opti-QISNet) and our recently developed fully self-supervised network models QIBDS Net [16], QIS-Net [17] and Opti-QIBDS Net [20]. The box plots are also demonstrated in 16 and 17 as the results reported in Table 3. Moreover, to show the effectiveness of our proposed Opti-QISNet over QIBDS Net, Opti-QIBDS Net, QIS-Net, Fuzzy C-means clustering, U-net and FCNNs, we have conducted one sided two sample Kolmogorov-Smirnov (KS) test with significance level $\alpha=0.05$. It is interesting to note that in spite being a fully self-supervised quantum learning inspired by qutrits, the Opti-QISNet has shown similar accuracy $(A C C)$ and dice similarity $(D S)$ compared to U-Net. Hence, it can be concluded, that the performance of the Opti-QISNet model on Dynamic Susceptibility Contrast (DSC) brain MR images is statistically significant and offers a potential alternative to the solution of deep learning technologies for medical image segmentation in near future.

\section{Conclusion}

A novel attempt has been made using an optimized version of the QuantumInspired Self-supervised Neural Network model encompassing the optimal thresholds using Otsu's method optimized by quantum-inspired meta-heuristics for fully automatic segmentation of brain MR images with minimum human intervention. Efficacy of the suggested Opti-QISNet model is demonstrated on the segmentation of Dynamic Susceptibility Contrast (DSC) MR images and compared with unsupervised fuzzy-C-means clustering (FCM), supervised convolutional neural network (CNN) based U-Net and fully convolutional neural network (FCNN) architectures and our recently developed quantum-inspired self-supervised neural network models (QIBDS Net, QIS Net, Opti-QIBDS Net). The optimal weights obtained during the self-supervision process outperforms quantum-inspired self-supervised models for brain MR image segmentation tasks. It may be noted that incorporation of optimal weights in the form of qubits using quantum-inspired meta-heuristics, leads to reduce the number of computation with the inputs and it enables faster convergence than the existing quantuminspired self-supervised models. In this paper, Opti-QIS-Net attains higher accuracy and significant dice similarity in spite being a fully self-supervised neural network model. However, the current Opti-QISNet model is applied in slice-wise computation and fails to capture the semantic features from the 3D

MR images. Authors are currently engaged in developing the $3 \mathrm{D}$ version of the self-supervised network architecture for volumetric brain MR segmentation. 

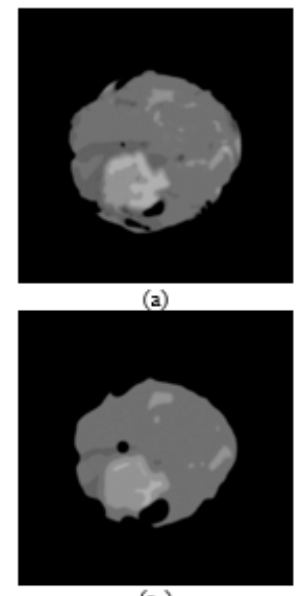

(a)

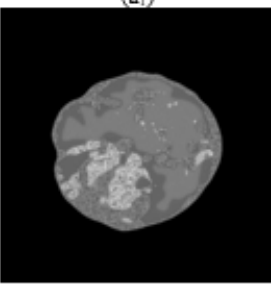

(a)

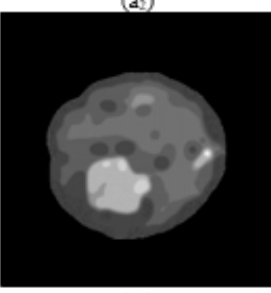

(a3)

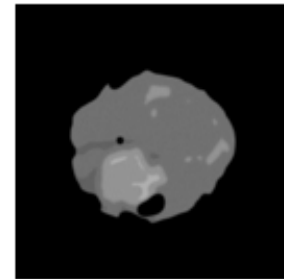

(b)

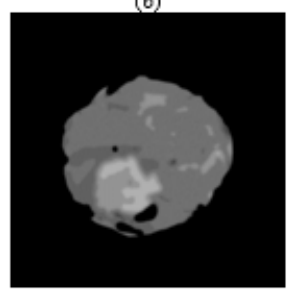

(b)



(b2)

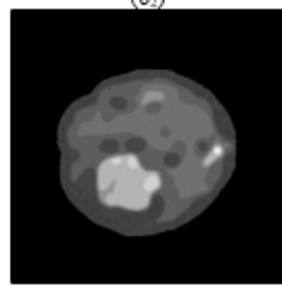

(b)



(c)

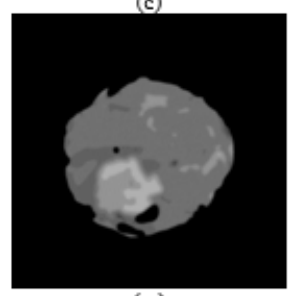

(c)

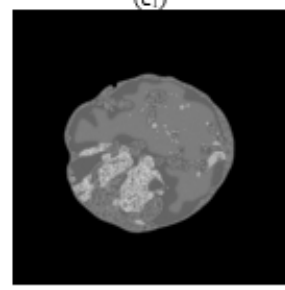

(c)

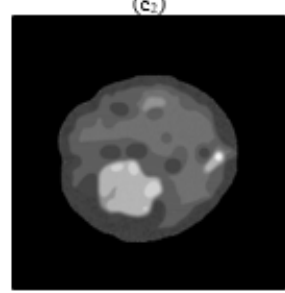

(c)

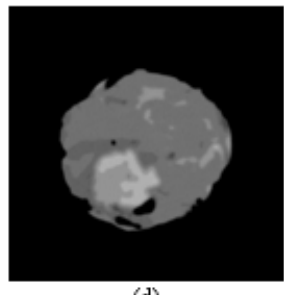

(d)

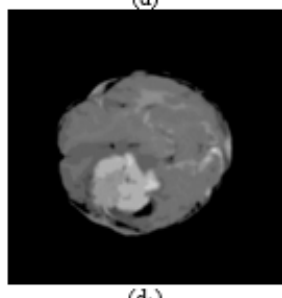

(d)

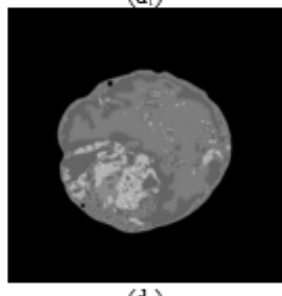

(d)

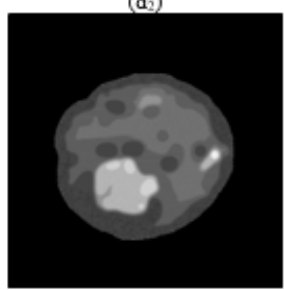

(d)

Figure 9: Segmentation using QDE-QISNet for slice \#3 with $\mathcal{L}=6$ (Activation $\left(a-a_{3}\right) \eta_{\beta},\left(b-b_{3}\right) \eta_{\chi},\left(c-c_{3}\right) \eta_{\xi},\left(d-d_{3}\right) \eta_{\nu}$. Level set $(a-d) \lambda_{1},\left(a_{1}-d_{1}\right) \lambda_{2}$, $\left.\left(a_{2}-d_{2}\right) \lambda_{3},\left(a_{3}-d_{3}\right) \lambda_{4}\right)$ 




(a)

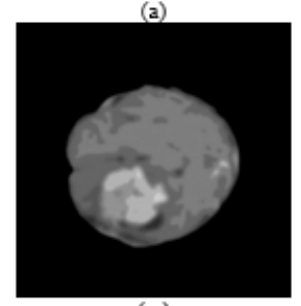

(a)

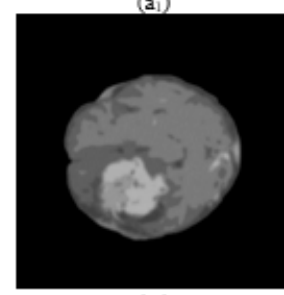

(a)

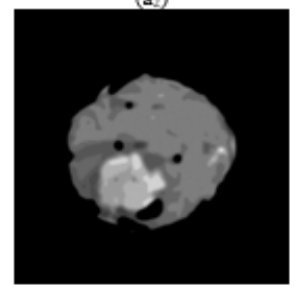

(a)

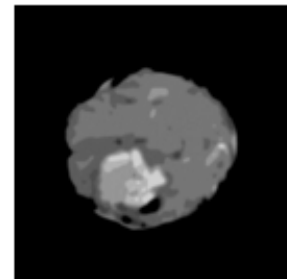

(b)

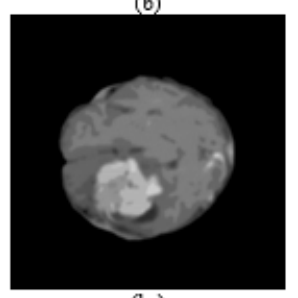

(b)

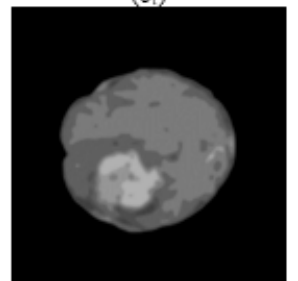

(b)

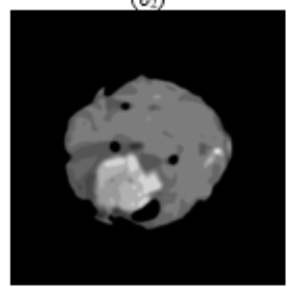

(b3)

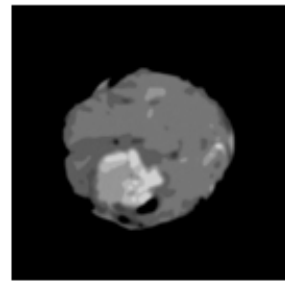

(c)

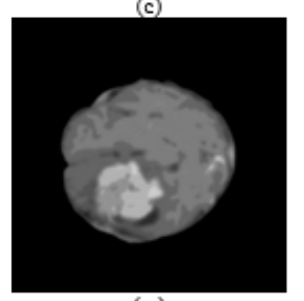

(c)

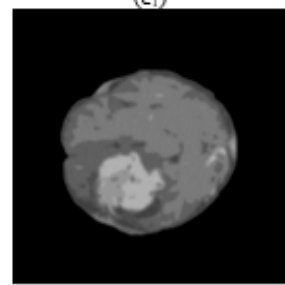

(c2)

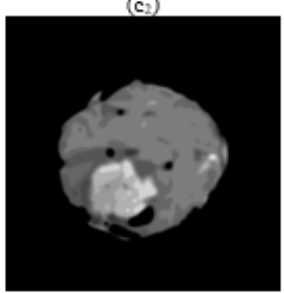

(c)

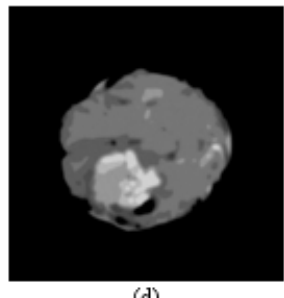

(d)

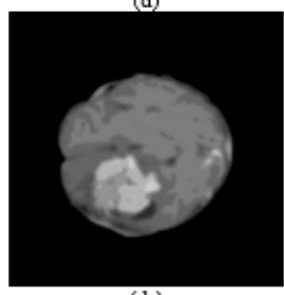

(d)

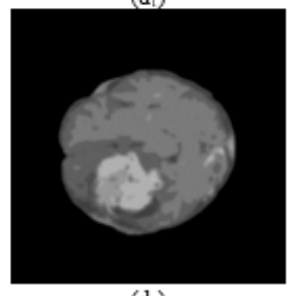

(d)

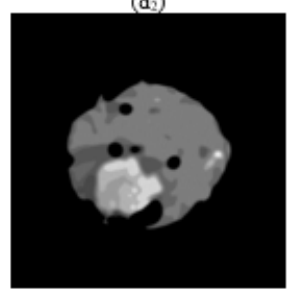

(d)

Figure 10: Segmentation using QDE-QISNet for slice \#3 with $\mathcal{L}=8$ (Activation $\left(a-a_{3}\right) \eta_{\beta},\left(b-b_{3}\right) \eta_{\chi},\left(c-c_{3}\right) \eta_{\xi},\left(d-d_{3}\right) \eta_{\nu}$. Level set $(a-d) \lambda_{1},\left(a_{1}-d_{1}\right) \lambda_{2}$, $\left.\left(a_{2}-d_{2}\right) \lambda_{3},\left(a_{3}-d_{3}\right) \lambda_{4}\right)$ 


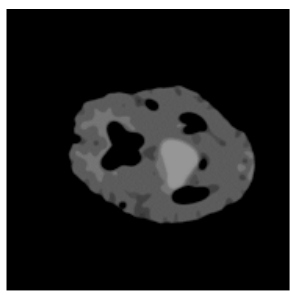

(a)

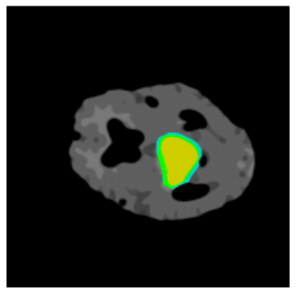

(a1)

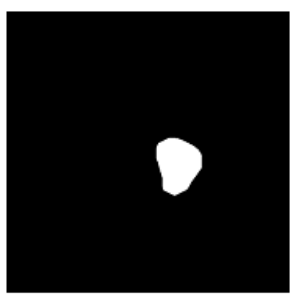

(a)



(b)

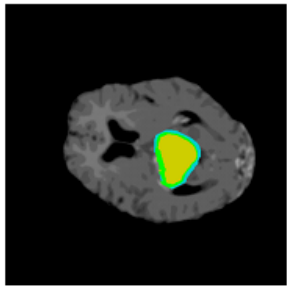

(b1)

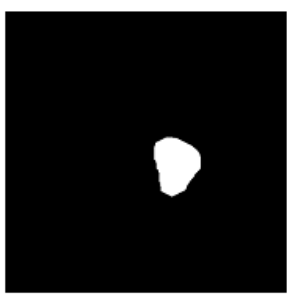

$\left(b_{2}\right)$

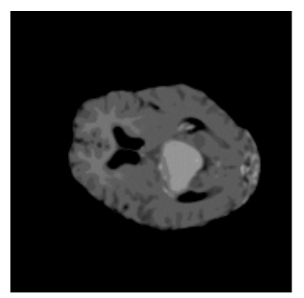

(c)



(c1)

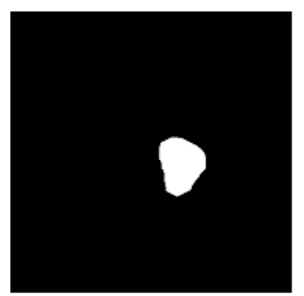

(c2)

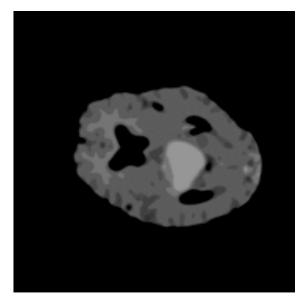

(d)

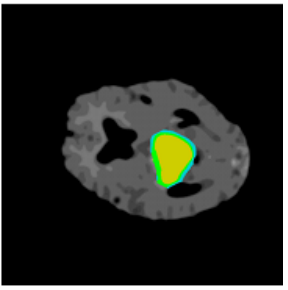

(d)

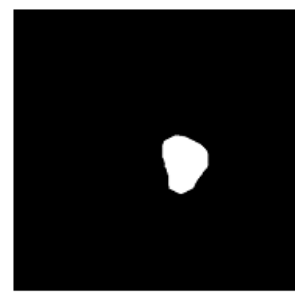

(d)

Figure 11: $(a-d)$ Segmentation using QDE-QISNet, $\left(a_{1}-d_{1}\right)$ Post processed with color map and $\left(a_{2}-d_{2}\right)$ Complete tumor segmentation on slice \#21 with $\mathcal{L}=8$ (Activation $\eta_{\beta}\left(a-a_{2}\right), \eta_{\chi}\left(b-b_{2}\right), \eta_{\xi}\left(c-c_{2}\right)$ and $\left.\eta_{\nu}\left(d-d_{2}\right)\right)$. 


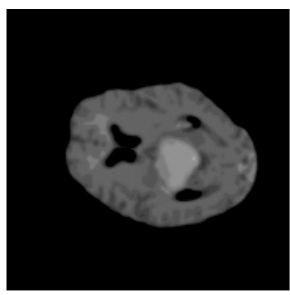

(a)

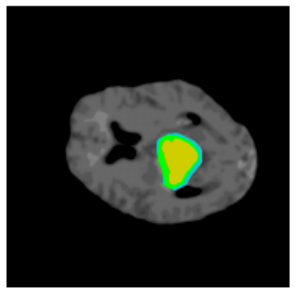

(a1)

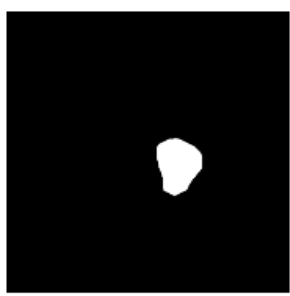

(a2)



(b)

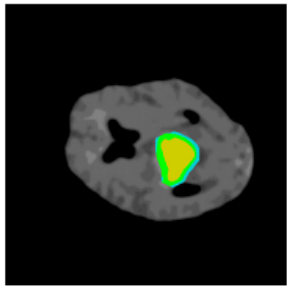

(b1)

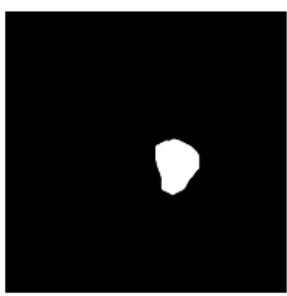

(b2)

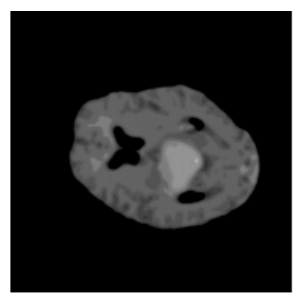

(c)

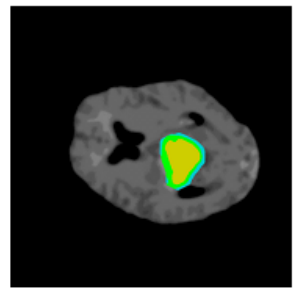

(c1)

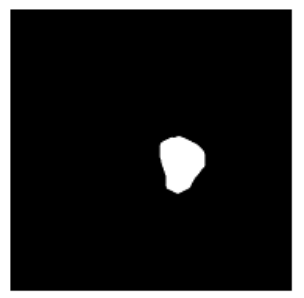

(c2)

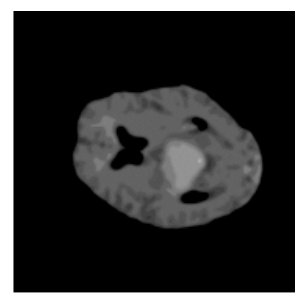

(d)

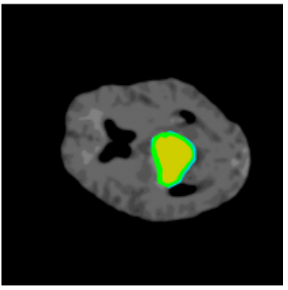

(d)



(d)

Figure 12: $(a-d)$ Segmentation using QACO-QISNet, $\left(a_{1}-d_{1}\right)$ Post processed with color map and $\left(a_{2}-d_{2}\right)$ Complete tumor segmentation on slice \#21 with $\mathcal{L}=8$ (Activation $\eta_{\beta}\left(a-a_{2}\right), \eta_{\chi}\left(b-b_{2}\right), \eta_{\xi}\left(c-c_{2}\right)$ and $\left.\eta_{\nu}\left(d-d_{2}\right)\right)$. 


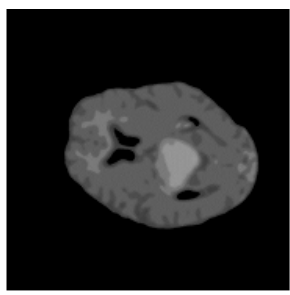

(a)

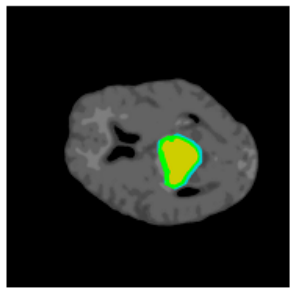

(a1)

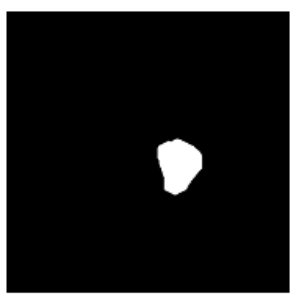

(a2)

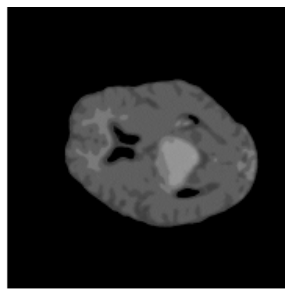

(b)

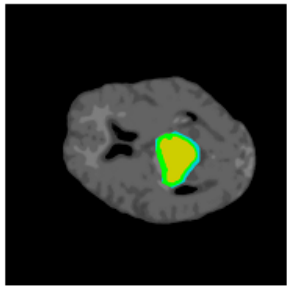

(b1)

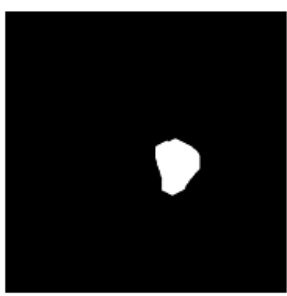

(b2)

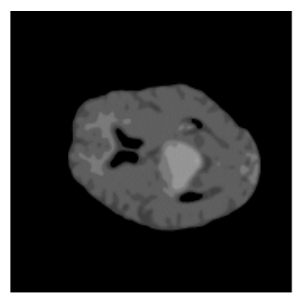

(c)

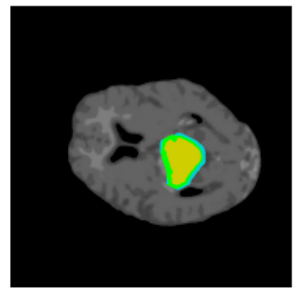

(c1)

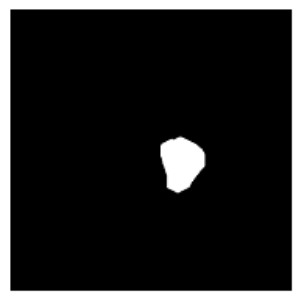

(c)

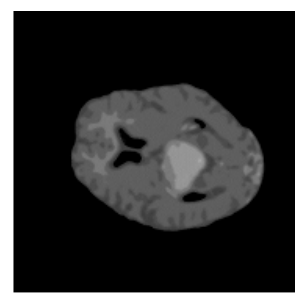

(d)

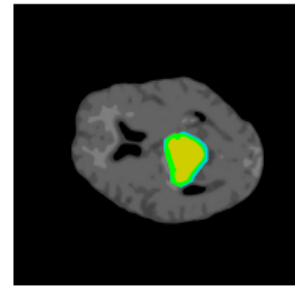

(d)

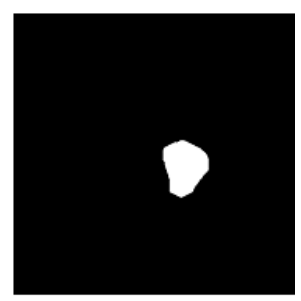

(d)

Figure 13: $(a-d)$ Segmentation using QPSO-QISNet, $\left(a_{1}-d_{1}\right)$ Post processed with color map and $\left(a_{2}-d_{2}\right)$ Complete tumor segmentation on slice \#21 with $\mathcal{L}=8$ (Activation $\eta_{\beta}\left(a-a_{2}\right), \eta_{\chi}\left(b-b_{2}\right), \eta_{\xi}\left(c-c_{2}\right)$ and $\left.\eta_{\nu}\left(d-d_{2}\right)\right)$. 


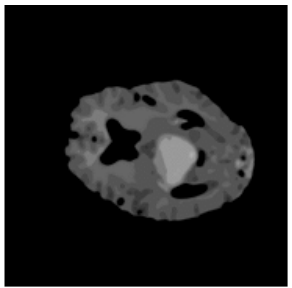

(a)

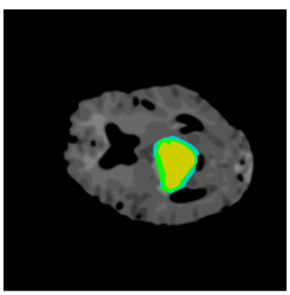

(a1)

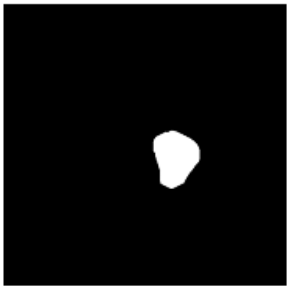

(a)

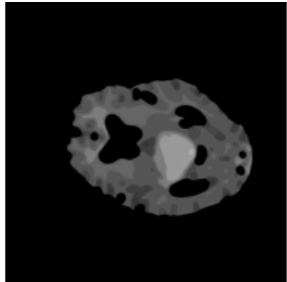

(b)



$\left(b_{1}\right)$

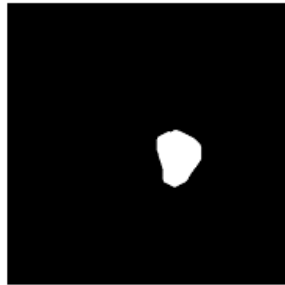

$\left(b_{2}\right)$

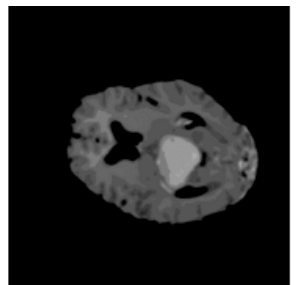

(c)

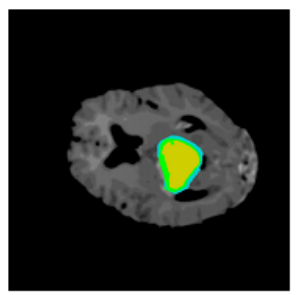

$\left(c_{1}\right)$

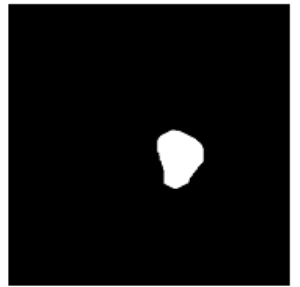

$\left(\mathrm{c}_{2}\right)$

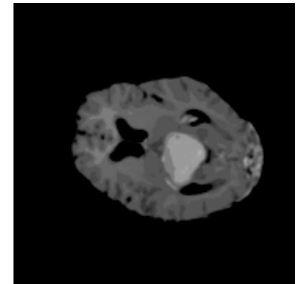

(d)

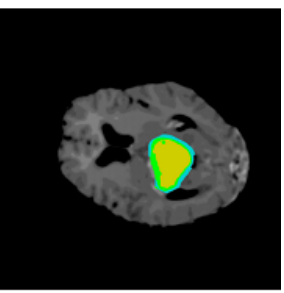

$\left(d_{1}\right)$



$\left(\mathrm{d}_{2}\right)$

Figure 14: $(a-d)$ Segmentation using QISNet, $\left(a_{1}-d_{1}\right)$ Post processed with color map and $\left(a_{2}-d_{2}\right)$ Complete tumor segmentation on slice \#21 with $\mathcal{L}=8$ (Activation $\eta_{\beta}\left(a-a_{2}\right), \eta_{\chi}\left(b-b_{2}\right), \eta_{\xi}\left(c-c_{2}\right)$ and $\left.\eta_{\nu}\left(d-d_{2}\right)\right)$.



(a)

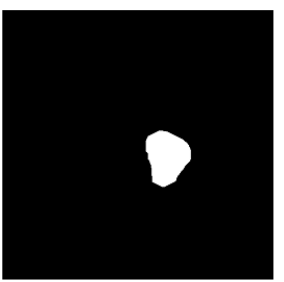

(b)

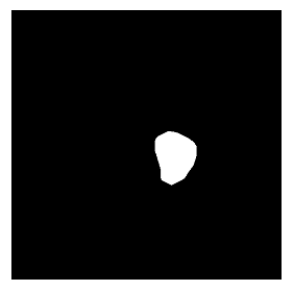

(c)

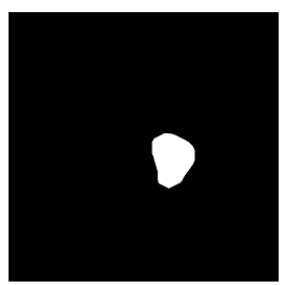

(d)

Figure 15: Segmented output images followed by post processing using (a) FCM [2] (b) CNN [28] (c) FCNN-2 [56] and (d) FCNN-4 [56] from slice \#21. 


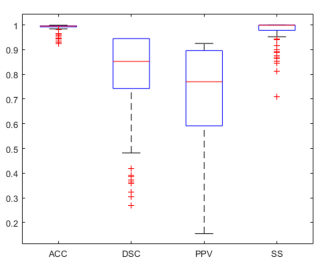

(a) QDE-QISNet, $\eta_{\beta}$

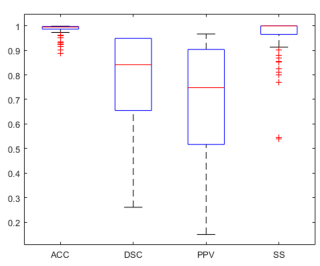

(d) QDE-QISNet, $\eta_{\nu}$

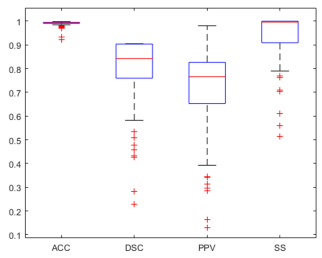

(g) QACO-QISNet, $\eta_{\xi}$

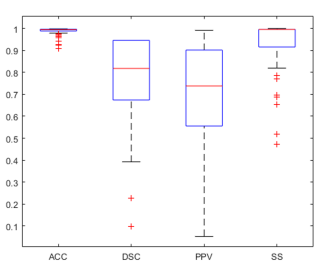

(j) QPSO-QISNet, $\eta_{\chi}$

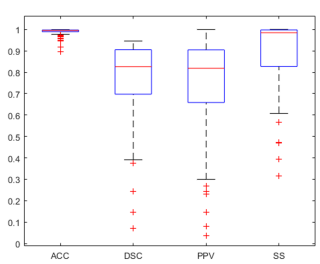

(m) QISNet, $\eta_{\beta}$

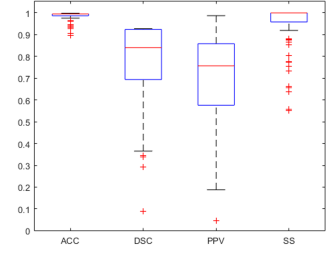

(b) QDE-QISNet, $\eta_{\chi}$

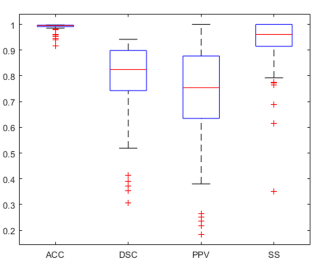

(e) QACO-QISNet, $\eta_{\beta}$

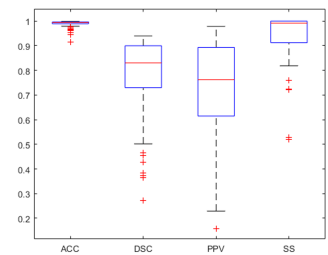

(h) QACO-QISNet, $\eta_{\nu}$

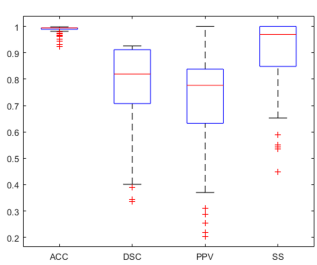

(k) QPSO-QISNet, $\eta_{\xi}$

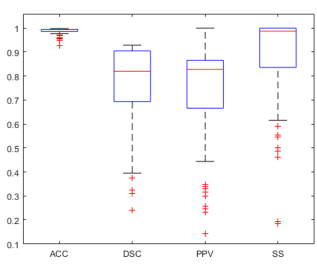

(n) QISNet, $\eta_{\chi}$

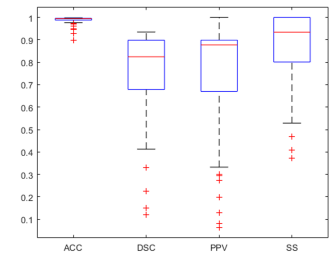

(p) QPSNet, $\eta_{\nu}$



(c) QDE-QISNet, $\eta_{\xi}$

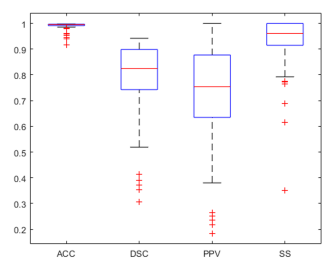

(f) QACO-QISNet, $\eta_{\chi}$

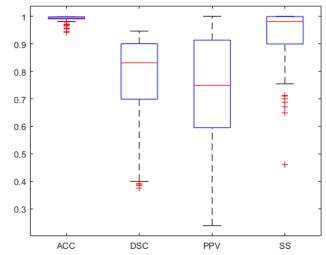

(i) QPSO-QISNet, $\eta_{\beta}$

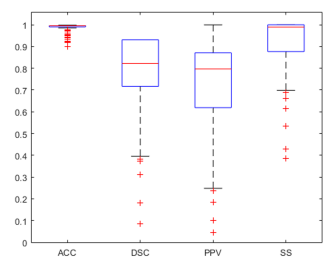

(l) QPSO-QISNet, $\eta_{\nu}$

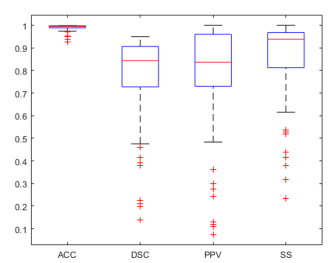

(o) QISNet, $\eta_{\xi}$

16: Box plot using $(a-d)$ QDE-QISNet, $(e-h)$ QACO-QISNet, $(i-l)$ QPSO-QISNet and $(m-p)$ QISNet [17], respectively for four different activation as reported in Table 3. 


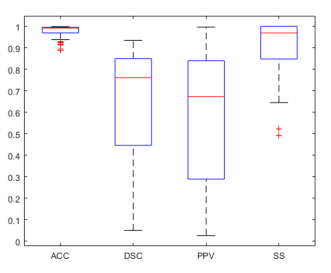

(a) FCM

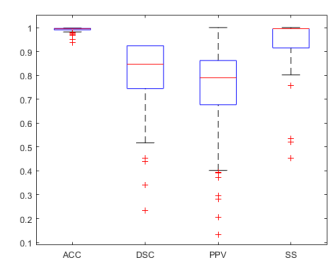

(b) U-Net



(d) FCNN-4

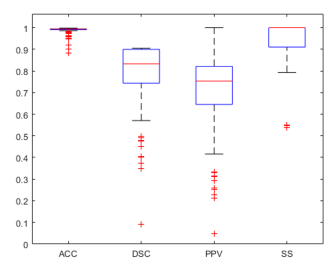

(c) FCNN-2

Figure 17: Box plot for $(q)$ FCM, $(r)$ U-Net, $(s)$ FNN-2 and $(t)$ FCNN-4, respectively as reported in Table 3 .

Finally, it can be confabulated from the proposed quantum-inspired and optimized self-supervised learning model that self-supervised learning paradigms sheds some light to become the potential alternatives to the realm of deep learning in near future.

\section{Conflicts of interest/Competing interests}

The authors hereby declare that there is no conflict of financial/personal interest or belief that could affect their objectivity.

\section{Code availability}

Pseudo code may be available after the final acceptance.

\section{References}

[1] L. M. Fletcher-Heath, L. O. Hall, D. B. Goldgof, F. R. Murtagh, Automatic segmentation of non-enhancing brain tumors in magnetic resonance images, Artif. Intell.Med. 21 (1-3) (2001) 43-63.

[2] H. Huang, F. Meng, S. Zhou, F. Jiang, G. Manogaran, Brain image segmentation based on fcm clustering algorithm and rough set, Special Section on New Trends in Brain Signal processing and Analysis: IEEE Access (2019) 12386-12396doi:10.1109/ACCESS. 2019.2893063. 
[3] S. Yazdani, R. Yusof, A. Karimian, M. Pashna, A. Hematian, Image segmentation methods and applications in mri brain images, IETE Technical Review 32 (2) (2015) 413-427.

[4] G. Litjens, T. Kooi, B. E. Bejnordi, A. A. Setio, F. Ciompi, M. Ghafoorian, J. A. . Laak, B. G. C. I. Sánchez, A survey on deep learning in medical image analysis, Medical Image Analysis 42 (2) (2017) 60-88.

[5] M. A. Nielson, I. L. Chung, Quantum computation and quantum information, Cambridge University Press, 2000.

[6] C. Chen, D. Dong, H. X. Li, J. Chu, T. J. Tarn, Fidelity-based probabilistic q-learning for control of quantum systems, IEEE Transaction on Neural Network and Learning Systems 25 (5) (2014) 920-933.

[7] L. K. Grover, A fast quantum mechanical algorithm for database search, In Proc. 28th ACM Symposium on Theory of Computing (1996) 212-219.

[8] P. Li, H. Xiao, F. Shang, X. Tong, X. Li, M. Cao, A hybrid quantuminspired neural networks with sequence inputs, Neurocomputing 117 (2013) $81-90$.

[9] S. Bhattacharyya, P. Pal, S. Bhowmik, A quantum multilayer self organizing neural network for object extraction from a noisy background, Proc. Fourth International Conference on Communication Systems and Network Technologies 117 (2014) 512-518.

[10] T. C. Lu, G. R. Yu, J. C. Juang, Quantum-based algorithm for optimizing artificial neural networks, IEEE Transaction on Neural Network and Learning Systems 24 (8) (2013) 1266-1278.

[11] S. Bhattacharyya, P. Pal, S. Bhowmick, Binary image denoising using a quantum multilayer self organizing neural network, Applied Soft Computing 24 (2014) 717-729.

[12] D. Konar, S. Bhattacharya, B. K. Panigrahi, K. Nakamatsu, A quantum bidirectional self-organizing neural network (qbdsonn) architecture for binary object extraction from a noisy perspective, Applied Soft Computing 46 (2016) 731-752.

[13] D. Konar, S. Bhattacharya, N. Das, B. K. Panigrahi, A quantum bidirectional self-organizing neural network (qbdsonn) for binary image denoising, Proc. IEEE International Conference on Advances in Computing and Communications and Informatics (ICACCI),2015 (2015) 1225-1230.

[14] D. Konar, S. Bhattacharya, U. Chakraborty, T. K.Gandhi, B. K. Panigrahi, A quantum parallel bi-directional self-organizing neural network (qpbdsonn) architecture for extraction of pure color objects from noisy background, Proc. IEEE International Conference on Advances in Computing and Communications and Informatics (ICACCI),2016 (2016) 1912-1918. 
[15] D. Konar, S. Bhattacharya, B. K. Panigrahi, M. K. Ghose, An efficient pure color image denoising using quantum parallel bidirectional self-organizing neural network architecture, Quantum Inspired Computational Intelligence (2016) 149-205.

[16] D. Konar, S. Bhattacharyya, B. K. Panigrahi, Qibds net: A quantuminspired bi-directional self-supervised neural network architecture for automatic brain mr image segmentation, Proc. 8th International Conference on Pattern Recognition and Machine Intelligence (PReMI 2019) 11942 (2019) $87-95$.

[17] D. Konar, S. Bhattacharyya, T. K. Gandhi, B. K. Panigrahi, A quantuminspired self-supervised network model for automatic segmentation of brain mr images, Applied Soft Computing (2020). doi:https://doi.org/10. $1016 /$ j.asoc. 2020.106348 .

[18] N. Otsu, A threshold selection method from gray level histograms, IEEE Transactions on Systems and Man and Cybernetics 9 (1) (1979) 62-66.

[19] S. Dey, I. Saha, S. Bhattacharyya, U. Maulik, Multi-level thresholding using quantum inspired meta-heuristics, Knowledge-Based Systems 67 (2014) 373-400.

[20] D. Konar, S. Bhattacharyya, S. Dey, B. K. Panigrahi, Opti-qibds net: A quantum-inspired optimized bi-directional self-supervised neural network architecture for automatic brain mr image segmentation, Proc. 2019 IEEE Region 10 Conference (TENCON) (2019) 761-766.

[21] P. Georgiardis, D. Cavouras, I. Kalatzis, A. Daskalakis, G. C. Kagadis, M. Mala-mas, G. Nikifordis, E. Solomou, Non-linear least square feature transformations for improving the performance of probabilistic neural networks in classifying human brain tumors on mri, Computational Science and Its Applications -ICCSA (2007) 239-247.

[22] V. Kumar, J. Sachdeva, I. Gupta, N. Khandelwal, C. K. Ahuja, Classification of brain tumors using pca-ann, Proc. of World Congress on Information and Communication Technologies (WICT) (2011) 1079-1083.

[23] L. O. Hall, A. M. Bensaid, L. P. Clarke, R. P. Velthuizen, M. Silbiger, J. C. Bezdek, Non-linear least square feature transformations for improving the performance of probabilistic neural networks in classifying human brain tumors on mri, IEEE Transactions on Neural Networks 3 (5) (1992) 672 682.

[24] S. B. Li. X, M. Kabuka, Labeling of mr brain images using boolean neural network, IEEE Transaction on Medical Imaging 15 (1996) 628-638.

[25] D. Zikic, et al., Context sensitive classification forests for segmentation of brain tumor tissues, Med. Image Com put. Comput.-Assisted Intervention Conf.-Brain Tumor Segmentation Challenge (2012). 
[26] S. Bauer, T. Fejes, J. Siotboom, R. Wiest, L. P. Nolte, M. Reyes, Segmentation of brain tumor images based on integrated hierarchical classification and regularization, Med. Image Comput. Comput.-Assisted Intervention Conf.-Brain Tumor Segmentation Challenge, Nice, France (2012).

[27] A. Ortiz, J. M. Gorriz, J. Ramirez, D. S. Gonzalez, Mri brain image segmentation with supervised som and probability-based clustering method, New Challenges on Bio-inspired Applications (IWINAC 2011) 6687 (2011) $49-58$.

[28] O. Ronneberger, P. Fischer, T. Brox, U-net: Convolutional networks for biomedical image segmentation, In proc. International Conference on Medical image computing and computer-assisted intervention 11 (8) (2015) 234241.

[29] M. Havaei, et al., Brain tumor segmentation with deep neural networks, Medical Image Analysis 35 (2017) 18-31.

[30] J. Lai, H. Zhu, X. Ling, Segmentation of brain mr images by using fully convolutional network and gaussian mixture model with spatial constraints, Mathematical Problems in Engineering (2019). doi:https://doi .org/10. $1155 / 2019 / 4625371$.

[31] M. Lyksborg, et al., An ensemble of 2d convolutional neural networks for tumor segmentation, Image Analysis. New York: Springer (2015) 201-211.

[32] S. Pereira, A. P. V. Alves, C. A. Silva, Brain tumor segmentation using convolutional neural networks in mri images, IEEE Transactions on Medical Imaging 35 (5) (2016) 1240-1255.

[33] S. Kak, On quantum neural computing, Information Sciences 83 (1995) 143-160.

[34] G. Purushothaman, N. B. Karayiannis, Quantum neural networks (qnns): inherently fuzzy feedforward neural networks, IEEE Transactions on Neural Networks 8 (3) (1997) 679-693.

[35] N. Kouda, N. Matsui, H. Nishimura, Image compression by layered quantum neural networks, Neural Processing Letters 16 (1) (2002) 67-80.

[36] N. Matsui, M. Takai, H. Nishimura, A network model based on qubitlike neuron corresponding to quantum circuit, Electronics and Communications in Japan (Part III: Fundamental Electronic Science) 83 (10) (2000) 67-73.

[37] A. Ghosh, N. R. Pal, S. K. Pal, Self organization for object extraction using a multilayer neural network and fuzziness measures, IEEE Transactions on Fuzzy Systems 1 (1) (1993) 54-68. 
[38] S. Bhattacharyya, P. Dutta, U. Maulik, Binary object extraction using bidirectional self-organizing neural network (bdsonn) architecture with fuzzy context sensitive thresholding, Pattern Anal Application 10 (2007) 345360.

[39] S. Bhattacharyya, P. Dutta, U. Maulik, Wave probabilities and quantum entanglement, Neural Network World: International JOURNAL on Neural and Mass - Parallel Computing and Information Systems 18 (5) (2008) 401-406.

[40] D. Ventura, T. Martinez, An artificial neuron with quantum mechanical properties, Proc. Intl. Conf. Artificial Neural Networks and Genetic Algorithms (1997) 482-485.

[41] D. Mcmohan, Quantum Computing Explained, John Wiley \& Sons, Inc., Hoboken, New Jersey, 2008.

[42] R. P. Feynman, R. B. Leighton, M. Sands, The feynman lectures on physics, Addison-Wesley Publishing Company 3 (1965).

[43] K. Takahashi, M. Kurokawa, M. Hashimoto, Multi-layer quantum neural network controller trained by real-coded genetic algorithm, Neurocomputing 134 (2014) 159-164.

[44] M. Dorigo, V. Maniezzo, A. Colorni, The ant system: optimization by a colony of cooperating agents, IEEE Trans. Syst. Man Cybernet. - Part B 26 (1) (1996) 29-41.

[45] R. Parpinelli, H. Lopes, A. Freitas, Data mining with an ant colony optimization algorithm, IEEE Trans. Evol. Comput. 6 (4) (2002) 321-332.

[46] S. Dey, I. Saha, S. Bhattacharyya, U. Maulik, New quantum inspired metaheuristic techniques for multi-level colour image thresholding, Applied Soft Computing 46 (4) (2016) 677-702.

[47] S. Dey, I. Saha, S. Bhattacharyya, U. Maulik, Efficient quantum inspired meta-heuristics for multi-level true colour image thresholding, Applied Soft Computing 56 (4) (2017) 472-512.

[48] R. Storn, K. Price, Differential evolution - a simple and efficient heuristic for global optimization over continuous spaces, ournal of Global Optimization 11 (1997) 341-359.

[49] K. Kennedy, R. Eberhart, Particle swarm optimization, in: Proceedings of the ieee international conference on neural networks (icnn95), JOURNAL of Global Optimization 4 (1995) 1942-194.

[50] S. Bhattacharyya, P. Dutta, U. Maulik, Multilevel image segmentation with adaptive image context based thresholding, Applied Soft Computing 11 (1) (2011) 946-962. 
[51] S. Bhattacharyya, U. Maulik, Soft Computing For Image And Multimedia Data Processing, Springer, Germany, 2013.

[52] S. Bhattacharyya, P. Dutta, U. Maulik, A self supervised bi-directional neural network (bdsonn) architechture for object extraction guided by beta activation function and adaptive fuzzy context sensitive thresholding, Int. J. Intell. Technol. 4 (1) (2006) 345-365.

[53] L. J. Zhen, X. G. He, D. S. Huang, Super-linearly convergent bp learning algorithm for feed forward neural networks, Journal of Software, in Chinese 11 (8) (2000) 1094-1096.

[54] H. Kim, et al., Convergence analysis of optimization algorithms (2017). doi:arXiv:1707.01647v1.

[55] K. M. Schmainda, M. A. Prah, J. M. Connelly, S. D. Rand, Glioma dscmri perfusion data with standard imaging and rois, The Cancer Imaging Archive (2020). doi:10.7937/K9/TCIA.2016.5DI84Js8.

[56] Y. Wang, Z. Sun, C. Liu, W. Peng, J. Zhang, Mri image segmentation by fully convolutional networks, Proc. of 2016 International Conference on Mechatronics and Automation and China (2016) 1697-1702.

[57] L. R. Dice, Measures of the amount of ecologic association between species, Ecology 26 (3) (1945) 297-302. 
Table 2: Performance of the proposed Opti-QISNet model optimized by Quantuminspired Differential Evolution (QDE) for slice \#3

\begin{tabular}{|c|c|c|c|c|c|c|c|c|c|}
\hline \multirow{2}{*}{ Level } & \multirow{2}{*}{ Set } & \multicolumn{2}{|c|}{$\overline{A C C}$} & & & \multicolumn{3}{|c|}{ DSC } & \\
\hline & & $\eta_{\beta}$ & $\eta_{\chi}$ & $\eta_{\xi}$ & $\eta_{\nu}$ & $\eta_{\beta}$ & $\eta_{\chi}$ & $\eta_{\xi}$ & $\eta_{\nu}$ \\
\hline \multirow{4}{*}{$\mathcal{L}=4$} & $\lambda_{1}$ & 0.98 & 0.98 & 0.97 & 0.98 & 0.56 & 0.54 & 0.41 & 0.49 \\
\hline & $\lambda_{2}$ & 0.98 & 0.98 & 0.99 & 0.98 & 0.65 & 0.69 & 0.81 & 0.71 \\
\hline & $\lambda_{3}$ & 0.98 & 0.98 & 0.98 & 0.98 & 0.34 & 0.54 & 0.62 & 0.60 \\
\hline & $\lambda_{4}$ & 0.98 & 0.98 & 0.98 & 0.98 & 0.59 & 0.57 & 0.53 & 0.59 \\
\hline \multirow{4}{*}{$\mathcal{L}=6$} & $\lambda_{1}$ & 0.99 & 0.99 & 0.99 & 0.99 & 0.86 & 0.86 & 0.86 & 0.85 \\
\hline & $\lambda_{2}$ & 0.99 & 0.99 & 0.98 & 0.98 & 0.86 & 0.86 & 0.85 & 0.84 \\
\hline & $\lambda_{3}$ & 0.99 & 0.99 & 0.99 & 0.98 & 0.84 & 0.88 & 0.85 & 0.85 \\
\hline & $\lambda_{4}$ & 0.99 & 0.98 & 0.98 & 0.98 & 0.84 & 0.84 & 0.84 & 0.84 \\
\hline \multirow{4}{*}{$\mathcal{L}=8$} & $\lambda_{1}$ & 0.99 & 0.98 & 0.98 & 0.98 & 0.85 & 0.85 & 0.84 & 0.84 \\
\hline & $\lambda_{2}$ & 0.99 & 0.99 & 0.99 & 0.99 & 0.89 & 0.88 & 0.88 & 0.87 \\
\hline & $\lambda_{3}$ & 0.99 & 0.98 & 0.99 & 0.98 & 0.86 & 0.62 & 0.86 & 0.76 \\
\hline & $\lambda_{4}$ & 0.98 & 0.98 & 0.98 & 0.98 & 0.84 & 0.84 & 0.84 & 0.84 \\
\hline \multirow{2}{*}{ Level } & \multirow{2}{*}{ Set } & \multicolumn{2}{|c|}{ PPV } & & & \multicolumn{2}{|c|}{ SS } & & \\
\hline & & $\eta_{\beta}$ & $\eta_{\chi}$ & $\eta_{\xi}$ & $\eta_{\nu}$ & $\eta_{\beta}$ & $\eta_{\chi}$ & $\eta_{\xi}$ & $\eta_{\nu}$ \\
\hline \multirow{4}{*}{$\mathcal{L}=4$} & $\lambda_{1}$ & 0.99 & 0.99 & 0.99 & 0.99 & 0.39 & 0.37 & 0.46 & 0.32 \\
\hline & $\lambda_{2}$ & 0.98 & 0.98 & 0.99 & 0.99 & 0.48 & 0.53 & 0.69 & 0.55 \\
\hline & $\lambda_{3}$ & 0.99 & 0.99 & 0.99 & 0.99 & 0.21 & 0.37 & 0.45 & 0.43 \\
\hline & $\lambda_{4}$ & 0.99 & 0.99 & 0.99 & 0.99 & 42.8 & 40.0 & 0.36 & 42.0 \\
\hline \multirow{4}{*}{$\mathcal{L}=6$} & $\lambda_{1}$ & 0.75 & 0.75 & 0.73 & 0.74 & 0.99 & 0.99 & 0.99 & 0.99 \\
\hline & $\lambda_{2}$ & 0.76 & 0.75 & 0.73 & 0.73 & 0.99 & 0.99 & 0.99 & 0.99 \\
\hline & $\lambda_{3}$ & 0.73 & 0.79 & 0.74 & 0.74 & 0.99 & 0.99 & 0.99 & 0.99 \\
\hline & $\lambda_{4}$ & 0.72 & 0.73 & 0.72 & 0.73 & 0.99 & 0.99 & 0.99 & 0.99 \\
\hline \multirow{4}{*}{$\mathcal{L}=8$} & $\lambda_{1}$ & 0.75 & 0.74 & 0.73 & 0.73 & 0.99 & 0.99 & 0.99 & 0.99 \\
\hline & $\lambda_{2}$ & 0.80 & 0.79 & 0.78 & 0.78 & 0.99 & 0.99 & 0.99 & 0.99 \\
\hline & $\lambda_{3}$ & 0.51 & 0.75 & 0.75 & 0.62 & 0.99 & 0.99 & 0.99 & 0.99 \\
\hline & $\lambda_{4}$ & 0.73 & 0.73 & 0.73 & 0.73 & 0.99 & 0.99 & 0.99 & 0.99 \\
\hline
\end{tabular}


Table 3: Comparative analysis of proposed Opti-QISNet with QISNet, QIBDS Net, Opti-QIBDS Net, FCM, U-Net, FCNN-2 and FCNN-4 using three quantum-inspired meta-heuristics[The bold values sheds light to the KS-significant data]

\begin{tabular}{|c|c|c|c|c|c|}
\hline Method & Activation & $\mathrm{ACC}$ & DSC & PPV & SS \\
\hline \multirow{4}{*}{ QDE-QISNet } & $\eta_{\beta}$ & 0.988 & 0.784 & 0.692 & 0.974 \\
\hline & $\eta_{\chi}$ & 0.983 & 0.766 & 0.684 & 0.946 \\
\hline & $\eta_{\xi}$ & 0.984 & 0.762 & 0.688 & 0.939 \\
\hline & $\eta_{\nu}$ & 0.984 & 0.773 & 0.688 & 0.960 \\
\hline \multirow{4}{*}{ QACO-QISNet } & $\eta_{\beta}$ & 0.990 & 0.788 & 0.719 & 0.932 \\
\hline & $\eta_{\chi}$ & 0.989 & 0.780 & 0.768 & 0.866 \\
\hline & $\eta_{\xi}$ & 0.989 & 0.790 & 0.714 & 0.935 \\
\hline & $\eta_{\nu}$ & 0.989 & 0.782 & 0.704 & 0.946 \\
\hline \multirow{4}{*}{ QPSO-QISNet } & $\eta_{\beta}$ & 0.989 & 0.770 & 0.700 & 0.928 \\
\hline & $\eta_{\chi}$ & 0.986 & 0.770 & 0.697 & 0.938 \\
\hline & $\eta_{\xi}$ & 0.987 & 0.774 & 0.717 & 0.903 \\
\hline & $\eta_{\nu}$ & 0.985 & 0.765 & 0.710 & 0.914 \\
\hline \multirow{4}{*}{ QISNet [17] } & $\eta_{\beta}$ & 0.987 & 0.763 & 0.739 & 0.887 \\
\hline & $\eta_{\chi}$ & 0.987 & 0.764 & 0.743 & 0.879 \\
\hline & $\eta_{\xi}$ & 0.989 & 0.774 & 0.783 & 0.855 \\
\hline & $\eta_{\nu}$ & 0.985 & 0.761 & 0.748 & 0.872 \\
\hline \multirow{4}{*}{ QIBDS Net [16] } & $\eta_{\beta}$ & 0.984 & 0.763 & 0.665 & 0.954 \\
\hline & $\eta_{\chi}$ & 0.985 & 0.765 & 0.720 & 0.960 \\
\hline & $\eta_{\xi}$ & 0.980 & 0.765 & 0.656 & 0.927 \\
\hline & $\eta_{\nu}$ & 0.989 & 0.763 & 0.657 & 0.961 \\
\hline \multirow{4}{*}{ Opti-QIBDS Net [20] } & $\eta_{\beta}$ & 0.987 & 0.752 & 0.674 & 0.819 \\
\hline & $\eta_{\chi}$ & 0.986 & 0.758 & 0.683 & 0.955 \\
\hline & $\eta_{\xi}$ & 0.987 & 0.767 & 0.656 & 0.897 \\
\hline & $\eta_{\nu}$ & 0.990 & 0.770 & 0.683 & 0.876 \\
\hline FCM $[2]$ & & 0.982 & 0.697 & 0.512 & 0.924 \\
\hline U-Net $[28]$ & & 0.990 & 0.808 & 0.729 & 0.940 \\
\hline FCNN-2 [56] & & 0.985 & 0.773 & 0.691 & 0.943 \\
\hline FCNN-4 [56] & & 0.984 & 0.761 & 0.679 & 0.953 \\
\hline
\end{tabular}

